\title{
Energy losses during drop weight mechanical impacts with special reference to ignition of flammable atmospheres in nuclear decommissioning: theory and determination of experimental coefficients for impact analysis and prediction.
}

\author{
A.F. Averill ${ }^{1}$, J.M. Ingram, P.G. Holborn and P. Battersby \\ Hydrogen Hazards Unit. London South Bank University (LSBU), London SE1 0AA UK.
}

\begin{abstract}
The major purpose of this study is to provide a framework for determination of energy losses resulting from mechanical impacts of the kind that could occur during nuclear decommissioning of waste material. Measurements have been made of final translational and rotational velocities for impacts between projectiles of different length and a massive barrier. This enabled determination of experimental values of the impact coefficients and energy losses. It was found that the total energy losses could be accurately accounted for by the sum of those pertaining to the normal and tangential processes, thus indicating that these include any losses due to vibration. The results obtained clearly support an Amontons-Coulomb friction model and the previously held contention that there is a limiting value for the impulse ratio at low angles of barrier inclination. Although sliding surfaces are likely to be modified during impact, it is shown that any original contamination on the contacting surfaces results in a very large decrease in impulse ratio or friction coefficient. This represents an important finding in the context of mechanical ignition testing indicating that the state of the impact surfaces and their handling need to be taken into account. The difficulties in establishing appropriate values for the impact coefficients and dealing with the effect of mechanical vibrations on the energy losses are discussed and equations derived for determining the tangential and normal energy losses from known initial velocities.
\end{abstract}

Keywords: velocity-measurements; energy-loss; impact-coefficients; vibration; surfacecontamination

\section{Notation}

$\begin{array}{ll}d & \text { distance } \\ D & \text { distance from centre of gravity just after impact } \\ e & \text { kinematic coefficient of normal restitution } \\ e_{m} & \text { coefficient of moment restitution } \\ E & \text { kinetic energy } \\ E_{l} & \text { kinetic energy loss } \\ E_{r} & \text { retained kinetic energy } \\ f & \text { coefficient of Coulomb friction } \\ F & \text { force }\end{array}$

${ }^{1}$ Corresponding author. E-mail address averilla@1sbu.ac.uk 


$\begin{array}{ll}g & \text { acceleration of gravity } \\ h & \text { impact tip test drop height } \\ I & \text { mass moment of inertia before impact } \\ J & \text { mass moment of inertia just after impact } \\ k & \text { radius of gyration } \\ l & \text { length } \\ M & \text { moment impulse } \\ m & \text { mass } \\ p & \text { impulse over a subinterval of contact duration } \\ P & \text { impulse over entire interval of impact duration } \\ R & \text { radius of impact tip or distance } \\ v & \text { velocity just before impact } \\ V & \text { velocity during the contact period } \\ V & \text { velocity after impact } \\ W & \text { work done by an impulse component } \\ \alpha & \text { impact angle (defined in Fig. } 1) \\ \gamma & \text { slenderness coefficient } \\ \Delta & \text { defined by Eq. } 5 \\ \mu & \text { ratio of tangential to normal impulse component } \\ \mu_{c} & \text { critical ratio of tangential to normal impulse component } \\ \tau & \text { time - impact duration } \\ \Gamma & \text { defined by Eq. } 4 \\ \Phi & \text { defined by Eq. } 25 \\ \omega & \text { angular velocity just before impact } \\ \Omega & \text { angular velocity after impact } \\ & \end{array}$

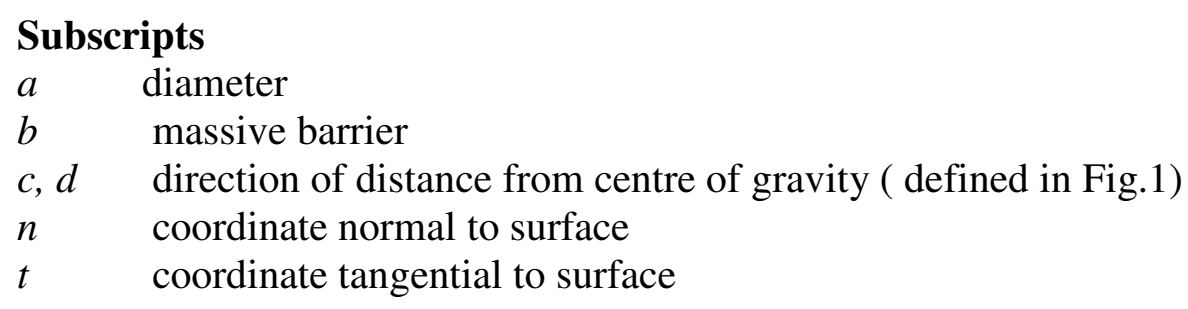

\section{Introduction}

Hydrogen explosion hazards have been recognised for many years in the nuclear industry, often in respect to loss of cooling incidents in power plant. They have also been a particular concern in relation to waste storage decommissioning and reprocessing operations with hydrogen produced by corrosion or radiolysis being held up in the waste sludge. Disturbance of this sludge together with possible mechanical impacts occurring during decommissioning could lead to the generation of ignition sources and deflagration. The major purpose of this study is to provide a framework for understanding and assessing the likely energy losses resulting from such mechanical impacts. Of most interest are the energy losses associated with tangential displacement between the contacting surfaces that result in a localised increase in temperature [1] sometimes sufficient to cause the ignition of a flammable atmosphere. Impacts can arise through 
movement of waste debris, failure of robotic arms or simply through accidentally dropped tools striking a barrier under the action of gravity. Ignition of flammable hydrogen atmospheres is readily caused by friction generated by clean metal surfaces sliding against each other where the mechanical loading and sliding velocity are sufficient to result in surface temperatures exceeding about $700^{\circ} \mathrm{C}$ for the necessary induction time period [2]. If pyrophoric substances such as Magnox-containing material from spent fuel cladding are present on the contacting surfaces, ignition becomes possible under much reduced loading conditions and sliding velocity [3]. The conditions necessary for ignition to occur when drop weight or glancing impacts are involved have also been investigated [4-6], indicating that contact surface temperatures lower than $500^{\circ} \mathrm{C}$ generated by impact can result in ignition when pyrophoric substances are present. To better understand the relevance of these studies it is necessary to have fuller knowledge of how energy is dissipated during such impacts, particularly those occurring during drop weight tests where the nature and source of the uncertainties is of considerable interest. It is also of importance to determine the effect of surface contamination on the manner in which energy is dispersed during impact.

In order to simplify the analysis of losses occurring over the entire contact period impulse-momentum methods were used. The assumption that impacts occur instantaneously (implicit in the impulse -momentum approach) poses few problems, since information relating to the dynamics occurring during the impact period is of much less interest than determination of the final translational and angular velocities. These velocities are required to establish the energy losses. As Brach [7] points out, the simplicity of classical impact theory is that it uses coefficients of restitution or friction (impulse ratio) to represent "the nasty behaviour that occurs at the interface in an impact". Although estimates of impact coefficients are often employed in predicting final velocities after an impact, appropriate experimental values are necessary for their proper evaluation and understanding. These coefficients are treated as constants in the system equations used to describe impact but there may be significant deviation from constancy in the real world owing to the influence of the material, surface condition and geometry of the impacting bodies as well as the initial velocities. To assess their usefulness in a practical context, it is thus necessary to determine experimental values and to explore their applicability over a range of impact angles and velocity.

In this paper, the relevant system equations relating to drop weight impacts are described (adopting Brach's [7] approach) and experimental results relating to impact velocities and coefficients presented. Disregarding the common assumption of point contact, Brach's more generalised concept of contact moment impulse is employed in the determination of final impact velocities and following from this the energy losses.

\subsection{System equations for drop weight impacts}

Painlevé $[8,9]$ highlighted a paradox in a simple rigid body contact problem (a planar box or rod rotating under gravity with its lower end contacting an horizontal rough surface) where a solution did not appear possible using rational impact mechanics and Admontons-Coulomb friction Law. If the friction coefficient is sufficiently large then before the contacting body separates and lifts off, it can assume a configuration (dynamic jam), indeterminate with respect to what follows. The possibilities are that the body either 
rebounds from the contacting surface or it rotates and digs into it. "It is as if there is a negative normal force pulling the tip into the surface" [9]. It follows that application of the friction law and Newton's Law of restitution becomes problematic since a so-called impact without collision may be indicated whereby an impulsive jump occurs to reduce the slip velocity of the tip to zero. Zhao et al. [10] have studied the Painlevé paradox at a slender uniform 3D rod and explained how a tangential stick occurs at the contact point during the impulsive process (where $f>4 / 3$ ). It should be noted, however, that under conditions where the mass is not uniform and concentrated near the centre of gravity the paradox can be shown to arise with low friction. Brach [7] dealt with the problem of improperly handling friction in collision problems by distinguishing the friction coefficient from the impulse ratio. In his impact model, a critical or limiting value of the impulse ratio $\left(\mu_{c}\right)$ exists that maximises the kinetic energy loss and which cannot be exceeded by any ascribed value of friction coefficient. This can be considered as a useful concept for many safety-case engineering applications in that $\mu_{c}$ is associated with the most pessimistic (i.e. largest) value of energy loss.

To characterise the amount of energy lost due to inelastic deformation during a collision, there are alternative definitions to the "kinematic" definition of the coefficient of restitution which relates the normal velocities of rebound and approach. The "kinetic" coefficient describes the ratio between the normal impulses for the restitution and compressive phases of the contact period. The "energetic" coefficient which relates the retrieved energy after impact to the initial energy has the advantage of being independent of the tangential impulse but leads to the considerable inconvenience of having to deal with non-linear equations.

For two-dimensional impact representation, Brach [7, 11, 12] made three assumptions in order to determine the final velocities after impact. (i) The coefficient of restitution is defined kinematically to include translational and rotational components: it represents the ratio in the normal direction of the final to initial (just before) impact velocities at the contact point or region. (ii) Ratio of the tangential to the normal impulse has a limiting value related to friction: there is a critical value $\mu_{c}$, dependent on the angle of incidence, beyond which sliding ceases. (iii) Contact forces between colliding bodies may be distributed throughout a contact region rather than at a point requiring the introduction of a moment and moment impulse at the region. The moment restitution coefficient $e_{m}$ indicates the presence of a moment and its corresponding impulse over the contact surfaces.

Impacts involving drop weight projectiles onto a massive barrier or anvil used in ignition studies [4] represent a special case in terms of impact dynamics. Because one of the two impacting bodies is fixed, with no or little freedom of movement, velocity change of this body is very small and can usually be neglected leading to a simpler solution. Momentum is not conserved during these collisions because momentum imparted to the barrier is lost through interaction with its surroundings: i.e. the projectile and anvil are not only interacting with each other. Consequently it is not possible to express a system equation for this case in terms of momentum conservation. However, since only the velocity changes relating to the drop weight projectile are involved it is possible to represent such impacts with just three system equations specified with coefficients of restitution $e$, impulse ratio $\mu$ and the moment restitution $e_{m}$. With reference to Fig. 1 


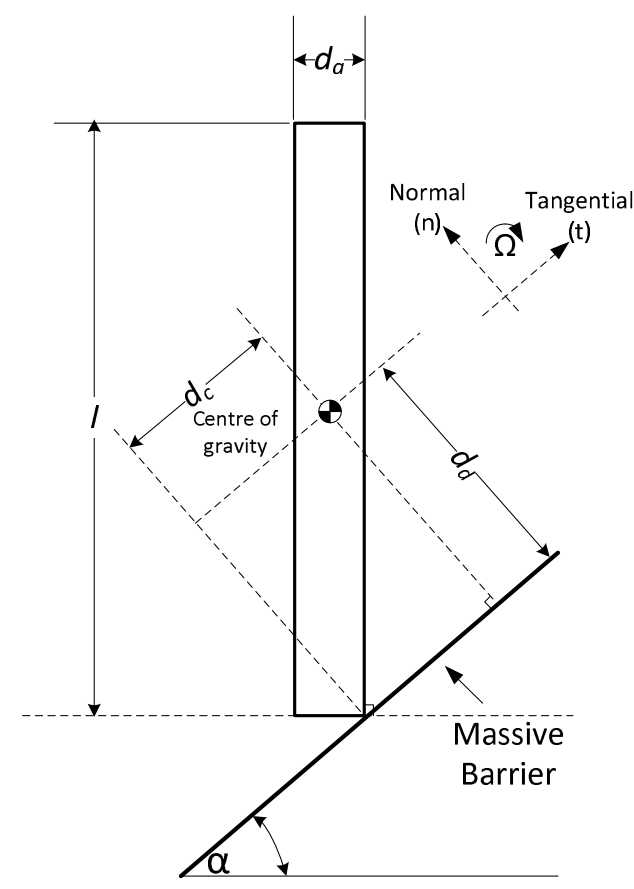

Figure 1. Impact between a falling projectile and inclined massive barrier

$$
\begin{aligned}
& V_{n}+D_{c} \Omega-v_{b n}=-e\left(v_{n}+d_{c} \omega-v_{b n}\right) \\
& \mu V_{n}-V_{t}=\mu v_{n}-v_{t}-\left(P_{1 t}-\mu P_{1 n}\right) / m \\
& e_{m} m \Gamma V_{n}-e_{m} m \Delta V_{t}+\left[1-e_{m}(J / I-1)\right] \Omega \\
& =e_{m}\left[m \Gamma v_{n}-m \Delta v_{t}-\omega+\Gamma P_{1 n}-\Delta P_{1 t}-M_{1} / I\right] \\
& \text { where } \Gamma=\frac{d_{c}+e D_{c}}{(1+e) I} \\
& \qquad \Delta=\frac{d_{d}+e D_{d}}{(1+e) I} \\
& d_{c}=0.5 \sqrt{l^{2}+d_{a}^{2}} \sin \left[\alpha-\pi / 2+\arctan \left(l / d_{a}\right)\right] \\
& d_{d}=0.5 \sqrt{l^{2}+d_{a}^{2}} \cos \left[\alpha-\pi / 2+\arctan \left(l / d_{a}\right)\right]
\end{aligned}
$$

Such linear system equations are easily solved for the unknown velocities $V_{n}, V_{t}$ and $\Omega$.

For convenient solution the coupled equations can be expressed in matrix form $\boldsymbol{A}$ with column vectors $\boldsymbol{b}$ and $\boldsymbol{x}$ : their elements are summarised in Table 1. 
$\boldsymbol{A} \boldsymbol{x}=\boldsymbol{b}$ where $\boldsymbol{A}=\left[\begin{array}{lll}\beta_{11} & \beta_{12} & \beta_{13} \\ \beta_{21} & \beta_{22} & \beta_{23} \\ \beta_{31} & \beta_{32} & \beta_{33}\end{array}\right] \boldsymbol{b}=\left[\begin{array}{c}\beta_{14} \\ \beta_{24} \\ \beta_{34}\end{array}\right]$ and $\boldsymbol{x}=\left[\begin{array}{c}V n \\ V t \\ \Omega\end{array}\right]$

Solutions are then readily obtained taking the minors of the determinants.

\begin{tabular}{|l|l|}
\hline \multicolumn{2}{|c|}{ Table 1. Matrix and vector elements for general case of impacts with massive barrier } \\
\hline$\beta_{11}$ & 1 \\
\hline$\beta_{12}$ & 0 \\
\hline$\beta_{13}$ & $D_{c}$ \\
\hline$\beta_{14}$ & $-v_{b n}+e\left(v_{n}+d_{c} \omega-v_{b n}\right)$ \\
\hline$\beta_{21}$ & $\mu$ \\
\hline$\beta_{22}$ & -1 \\
\hline$\beta_{23}$ & 0 \\
\hline$\beta_{24}$ & $-\mu v_{n}+v_{t}+\left(P_{1 t}-\mu P_{1 n}\right) / m$ \\
\hline$\beta_{31}$ & $e_{m} m \Gamma$ \\
\hline$\beta_{32}$ & $-e_{m} m \Delta$ \\
\hline$\beta_{33}$ & $1-e_{m}(J / I-1)$ \\
\hline$\beta_{34}$ & $-e_{m}\left[m \Gamma v_{n}-m \Delta v_{t}-\omega+\Gamma P_{1 n}-\Delta P_{1 t}-M_{1} / I\right]$ \\
\hline
\end{tabular}

Relating to drop weight experimental tests, a number of assumptions can be made to further simplify Eq. $1-3$. The only external impulse (i.e. those which would still exist when the bodies are not in contact denoted by suffix 1) that cannot be effectively eliminated in experimental tests is that due to gravity. However, for these impacts, its effect can be disregarded. If a large massive barrier is used then $v_{b n}$ can be taken as zero as of course can the initial angular velocity $\omega$ for vertically dropped projectiles. It is further assumed that change in shape of the projectile will be insignificant so that $J=I$, $D_{c}=d_{c}$ and $D_{d}=d_{d}$. Under these conditions the system equations reduce to

$$
\begin{aligned}
& V_{n}+d_{c} \Omega+e v_{n}=0 \\
& \mu\left(V_{n}-v_{n}\right)-V_{t}+v_{t}=0
\end{aligned}
$$

If tangential motion ceases at or prior to separation, then Eq. 10 is replaced by the simple condition

$$
V_{t}-d_{d} \Omega=0
$$

corresponding to a critical value $\mu_{c}$ of the impulse ratio.

Finally

$$
\left(e_{m} m / I\right)\left[d_{c}\left(V_{n}-v_{n}\right)-d_{d}\left(V_{t}-v_{t}\right)\right]+\Omega=0
$$

In this case the moment restitution coefficient for impact onto a massive barrier is defined by the expression 


$$
e_{m} M=\left(1+e_{m}\right) I \Omega
$$

Because of the independence of translational and rotational motion, the tip velocities after impact are easily determined taking into account the centre of mass translational velocities and the angular velocity

$$
\begin{aligned}
& V_{c t}=V_{t}-d_{d} \Omega \\
& V_{c n}=V_{n}+d_{c} \Omega
\end{aligned}
$$

The impact coefficients are obtained from experimentally determined velocities

$$
\begin{gathered}
e=-\frac{\left(V_{n}+d_{c} \Omega\right)}{v_{n}} \\
\mu=\frac{V_{t}-v_{t}}{V_{n}-v_{n}} \\
e_{m}=-\frac{k^{2} \Omega}{d_{c}\left(V_{n}-v_{n}\right)-d_{d}\left(V_{t}-v_{t}\right)}
\end{gathered}
$$

It is seen from Eq.13 and 18 that the value of the moment restitution coefficient effectively represents the nature of the rotational kinematics as well as the distribution of forces in the contact region. When $e_{m}=-1$, the moment impulse $M=0$ and the planar forces are effectively represented as point forces at a given point. On the other hand, $e_{m}$ $=0$ implies that $\Omega=0$ and is indicative of a perfectly inelastic angular impact. It is less readily seen (but can be shown [7]) that $e_{m}=1$ corresponds to an impact that is perfectly elastic.

\subsection{Determination of energy losses}

Energy losses during an impact are related to the work done by the impulses where the partial work done by each component of impulse is equivalent to the change in kinetic energy brought about by the contact forces. It is intuitive that energy losses on impact can be expressed in terms of impulse components and average of the velocity change; this follows since work is the time integral of power (i.e. $F_{i} \times v_{i}$ component).

$$
W_{i}=\int_{0}^{\tau} F_{i} v_{i} d \tau=\int_{0}^{P} v_{i} d P_{i}
$$

The partial work done on impact by either the normal or tangential components of impulse is given by the scalar product of the impulse component and half (i.e. average) of the sum of the respective initial and final velocity components.

The normal and tangential energy losses can thus be shown respectively to be

$$
\begin{aligned}
& E_{\mathrm{ln}}=0.5 m\left[\left(v_{n}^{2}-V_{n}^{2}+\Omega d_{c}\left(v_{n}-V_{n}\right)\right]\right. \\
& E_{l t}=0.5 m\left[\left(v_{t}^{2}-V_{t}^{2}-\Omega d_{d}\left(v_{t}-V_{t}\right)\right]\right.
\end{aligned}
$$


However, as noted by Stronge [13], Brach [7] and others, anomalies will arise if there is any change of direction during impact. This can give rise to apparent (erroneous) gains rather than losses of energy so that when contact velocities are not unidirectional, it would be necessary to split the overall contact period into discrete periods of unidirectional sliding. Brach [12] considers arbitrary subintervals of time within the contact duration for eccentric impacts with a slender rod and computes changes in the impulse ratio and tip velocities when contact involves stop and reverse. An equation is given to determine $\mu$ over any sub interval of time $\left(\tau_{b}-\tau_{\mathrm{a}}\right)$, necessary for the determination of subinterval energy losses. Expressed in the notation of this paper:

$\mu(\Delta \tau)=\frac{\Delta p_{t}}{\Delta p_{n}}=\frac{\left(k^{2}+d_{c}^{2}\right)\left[v_{c t}\left(\tau_{b}\right)-v_{c t}\left(\tau_{a}\right)\right]+d_{c} d_{d}\left[v_{c n}\left(\tau_{b}\right)-v_{c n}\left(\tau_{a}\right)\right]}{\left(k^{2}+d_{d}^{2}\right)\left[v_{c n}\left(\tau_{b}\right)-v_{c n}\left(\tau_{a}\right)\right]+d_{c} d_{d}\left[v_{c t}\left(\tau_{b}\right)-v_{c t}\left(\tau_{a}\right)\right]}$

Assuming, however, that only unidirectional sliding (and possibly stopping) occurs and taking into account Eq. 9 -12 and 20-21, expressions for the energy losses can be derived in terms of the three impact coefficients with known initial velocities.

$$
E_{\mathrm{ln}}=\frac{0.5 m v_{n}^{2}}{1+\Phi}\left(1-e^{2}\right)
$$

and

$$
\begin{aligned}
E_{l t} & =0.5 m v_{n} \mu k^{-2}(1+e)\left[\mu v_{n}(e+1)\left(d_{d}^{2} e_{m}-k^{2}\right)+2 v_{t}\left(k^{2}-e_{m} d_{c}^{2}\right)\right. \\
& \left.+d_{c} d_{d} e_{m}\left(2 \mu v_{t}-(1+e) v_{n}\right)\right][1+\Phi]^{-2}
\end{aligned}
$$

where

$$
\Phi=e_{m} d_{c}\left(\mu d_{d}-d_{c}\right) / k^{2}
$$

Noting that the kinetic energy $\left(E_{r}\right)$ retained by the projectile after impact is given by the sum of the translational and rotational components

$$
E_{r}=0.5\left(I \Omega^{2}+m\left(V_{n}^{2}+V_{t}^{2}\right)\right)
$$

It follows that, if all of the energy loss after impact is accounted for by $E_{l t}$ and $E_{l n}$, then the sum of $E_{l t}, E_{l n}$ and $E_{r}$ should be similar to the initial impact energy.

\subsection{Method of experimentally determining impact coefficients and kinetic energy losses}

There are difficulties in releasing a body to fall vertically without imparting some degree of side thrust that initially alters its trajectory. The mechanism finally chosen is shown schematically in Fig. 2. A Perspex guide was carefully machined and the projectile body polished to allow the projectile to slide easily without side movement and without

lubrication. A slot was cut into the guide so that the projectile was held in close proximity 
(not touching) to a powerful electromagnet (9W 1400N holding force). Because the face of the magnet was vertical this ensured that when the current was switched off there was no sideways thrust or angular velocity imparted to the projectile. The electromagnet itself was attached rigidly to the supports so that the projectile could be held in the same position throughout a sequence of tests. With this arrangement, it was found that projectiles dropped from a height $h=0.82 \mathrm{~m}$ impacted the test plate with a position randomly displaced by no more than $\pm 2 \mathrm{~mm}$. The initial translational velocities on impact with a barrier inclined at an angle $\alpha$ (c.f. Fig. 1) are given by

$$
\begin{aligned}
& v_{n}=-\sqrt{2 g h} \cos \alpha \\
& v_{t}=-\sqrt{2 g h} \sin \alpha
\end{aligned}
$$

Three medium carbon steel projectiles were used in the study cut from $19 \mathrm{~mm}$ diameter rod with measured mechanical properties: YP $546 \mathrm{~N} / \mathrm{mm}^{2}$, UTS $592 \mathrm{~N} / \mathrm{mm}^{2}, \mathrm{E}=219$ $\mathrm{GPa}$ and hardness $235 \mathrm{HV}$. The first of these, of overall length $0.24 \mathrm{~m}$ had either a machined tip with a $7 \mathrm{~mm}$ radius hemispherical end (profile A) or a cut and machined faced end with a $3 \mathrm{~mm}$ radiused edge (profile B). Other projectiles, of lengths 0.12 and $0.08 \mathrm{~m}$ were similarly machined to achieve a tip with profile B. In all cases after impact there was some permanent damage to both the projectile tip and barrier plate necessitating renewal of the surfaces by further machining with clean lathe cutting tools. For each projectile, the centre of mass was found by balancing on a knife edge and a tracking marker attached. A second tracking marker was attached near to the tip to allow the determination of change in angular velocity after impact. This is shown in Fig 3 together with an image of a typical indentation made during impact.

The body of the main anvil with $\alpha=\pi / 4\left(45^{\circ}\right)$ was constructed from a large billet section of steel $(0.3 \mathrm{~m} \times 0.15 \mathrm{~m}$ diameter $)$ robustly welded onto a $12 \mathrm{~mm}$ thick steel plate securely bolted to the concrete floor. High speed video images confirmed that the movement of the barrier during impacts (i.e. $v_{b n}$ ) could be neglected even with impacting projectiles heavier than those employed in this study. To obtain measurements for lower velocity impacts with $\alpha \neq \pi / 4$ a variable angle steel barrier (also shown in Fig. 2) was used. Again, it was confirmed that insignificant movement of the barrier occurred during experimental impacts. Test plates of plain carbon steel (YP $320 \mathrm{~N} / \mathrm{mm}^{2}$, UTS 446 $\mathrm{N} / \mathrm{mm}^{2}, \mathrm{E}=206 \mathrm{GPa}$ and hardness $163 \mathrm{HV}$ ) which could be resurfaced and repositioned as required were tightly bolted onto either of the steel barriers. After machining, the surface profile condition was measured with a Taylor Hobson Surtronic 10 surface roughness profiler and a typical value of 3.9 obtained for $\mathrm{Ra}$ (average profile height deviation from the mean). In some cases, as noted in the text, both the projectile impact surfaces and anvil plates were carefully cleaned just before tests were carried out. This was accomplished in an ultrasonic bath with HFE 71DE (Methoxynonafluorobutane trans-1,2-Dichloroethylene 1:1 azeotrope) at $25^{\circ} \mathrm{C}$ for 10 minutes [14].

A Photron Fascam-1024PCL 100K high- speed camera was used to record the impact events, utilising a frame speed of 10,000 FPS at a resolution of 512 x 128 pixels. With suitable modification of the information files it was possible to use Olympus "Deluxe" i-Speed software to calibrate and analyse the video images with respect to final translational and rotational velocities; a typical tracking image for an impact is shown in 
Fig. 3. $V_{t}$ and $V_{n}$ were obtained by tracking the marker positioned at the centre of mass whilst the tip velocities $V_{c t}$ and $V_{c n}$ were determined from the movement of the lower marker. In order to determine rotational velocity it was necessary to consider the relative movement of both markers. In all cases, the final velocity values were determined from the mean of 40 frames (i.e. $4 \mathrm{~ms}$ ) immediately after separation. Direct comparison of calculated values of $v_{n}$ and $v_{t}$ with experimental values indicated that the error attributable to the tracking procedure was less than $2 \%$.
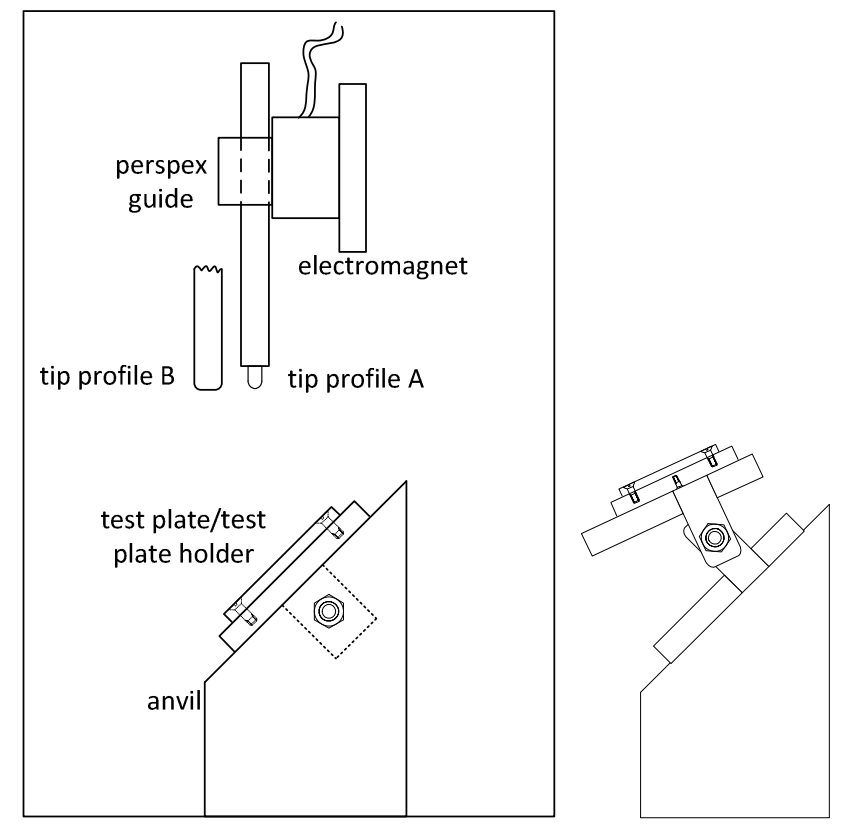

Figure 2. The projectile release mechanism with the fixed and low impact variable angle barriers.

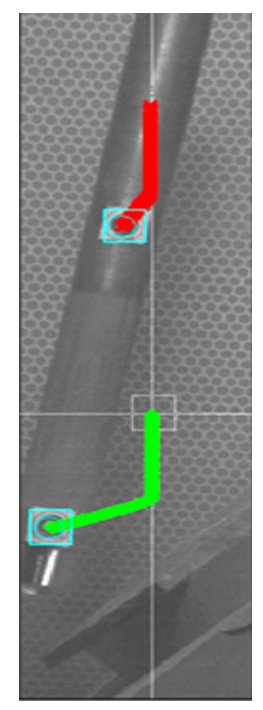

(a)

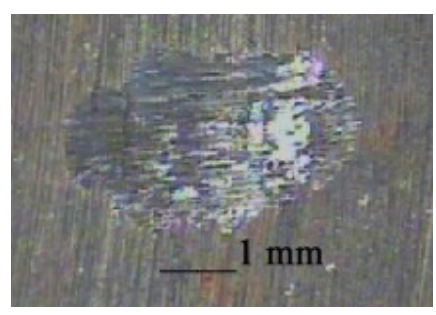

(b) 
Figure 3. (a) Typical high speed tracking image showing movement of the projectile centre of mass and near tip markers. (b) Typical impact indentation $(0.24 \mathrm{~m}$ projectile, tip profile A with impact velocity $3.9 \mathrm{~m} / \mathrm{s}$ ).

Although calculations of $d_{c}$ and $d_{d}$ can be made using Eq. 6 and 7, significant errors in determining the impact coefficients can arise if these do not properly reflect the geometry and impact point of the tip. Because the tip geometry affects both the centre of mass of the projectile and the exact location of the impact contact, calculated values of $d_{c}$ and $d_{d}$ for the $0.24 \mathrm{~m}$ tipped projectile were confirmed graphically from scale drawings of the projectile and anvil. This was of greatest importance for tips of profile A. Normal and tangential energy losses that occurred during impact were determined directly from the measured translational and angular velocities using Eq. 20 and 21. The KE retained by the projectile after impact was then determined from Eq. 26 so that direct comparison could be made between the sum of $E_{l n}, E_{l t}$ and $E_{r}$ and the initial impact energy.

\subsection{Experimental Results}

An initial set of experiments was carried out to explore the nature of the variation in the impact coefficients using tips and anvil plates that had been lightly handled. This corresponds to typical circumstances in the use of test materials in mechanical ignition testing of flammable gases where impacting surfaces may be contaminated by fingerprint residues and stored in the laboratory before use. The composition of bodily perspiration has been extensively studied for medical and forensic purposes. Girod et al [15] have described the composition of fingermark residues as a complex mixture of numerous compounds arising from three sources, dermis, eperdermis and extrinsic contaminants. The results of impact tests carried out with freshly produced finger residue contaminated surfaces are given in Table 2 . These clearly reveal the variation in the measured afterimpact velocities and the determined impact coefficients that can randomly arise from tests carried out under the same nominal experimental conditions. Bivariate plots of the impact coefficients (Fig. 4) show that there is no significant correlation between any of the coefficients indicating that their variation seems is quite independent. During the tests, considerable acoustic noise (longitudinal vibration) occurred on impact and clear indication of transverse vibration was observed in the digital imaging of the projectile motion after impact contact had ceased. In all the tests, the sum of the impact energy losses $E_{l n}$ and $E_{l t}$ together with the retained $\mathrm{KE}\left(E_{r}\right)$ show a close similarity with the initial impact energy. 

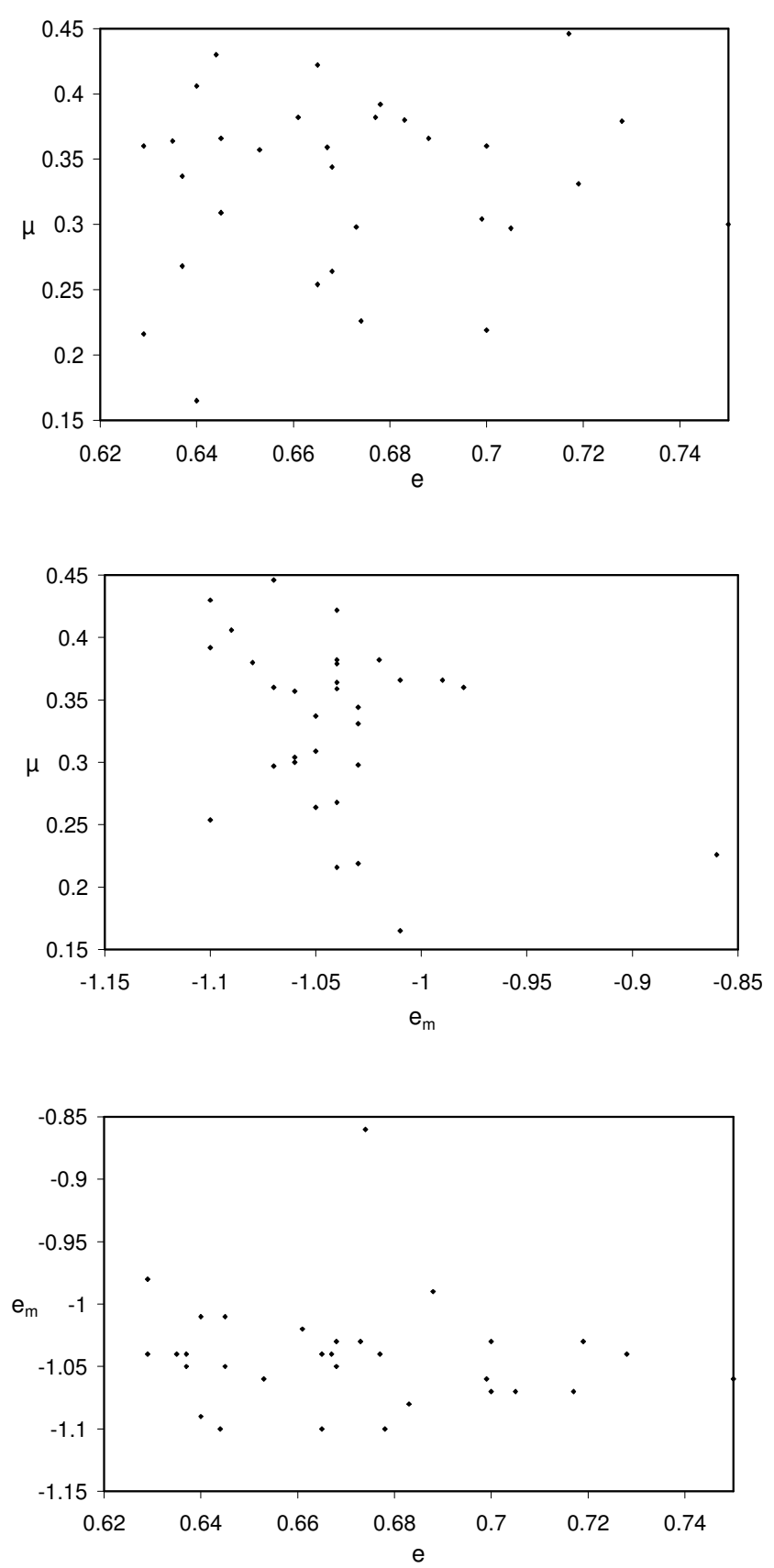

Figure 4. Bivariate plots of the impact coefficients from the repeated tests with steel on steel impacts.

To investigate the influence of surface cleanliness, a series of tests was carried out with different surface conditions: (i) freshly machined and uncontaminated (ii) fingermarked and (iii) cleaned. It can be seen from Table 3 that freshly machining the projectile and barrier impact surfaces had a pronounced effect on $\mu$, more than doubling its value when compared to the contaminated surface condition. A smaller but significant change in the magnitude of e can also be noted, with e being smaller for freshly machined rather than contaminated surfaces. Regardless of the surface condition, little variation in $e_{m}$ from a 
value of -1 was apparent indicating no change from zero angular impulse moment. Energy losses, determined from the measured velocities after impact, were considerably influenced by the surface condition, rising from around 50\% of the impact energy for the contaminated surfaces to more than $90 \%$ for those freshly machined.

Cleaning the contact tip with HFE 71DE after handling and surface contamination, whilst considerably increasing the impulse ratio did not completely restore the tip to the same condition as being freshly machined. The impulse ratios were slightly lower than for the freshly machined surfaces irrespective of whether wipe cleaning or ultrasonic cleaning was employed. At this stage, tests were also conducted to determine the effect of combustion flames on the impacting surfaces. Contaminated surfaces were subjected to several seconds of flaming from a portable propane torch before undergoing impacts under similar conditions to those reported in Table 3. No significant changes to the measured impact velocities and impact coefficients were recorded indicating that the surface contamination was not removed. All further experiments were carried out with freshly machined impact surfaces.

Acoustic noise and transverse vibration was found to be much less evident with impacts involving the shorter projectiles. In the case of the intermediate length projectile ( $0.12 \mathrm{~m}$ long), both $\mu$ and e values (Table 4 ) were significantly reduced when compared to the results obtained for the longer projectiles. As with the previous tests, the mean value for $e_{m}$ remains consistent with zero impulse moment and the determined energy losses can be accounted for in relation to the impact energy. Tests to investigate the effect of changing impact velocity and anvil barrier inclination were conducted using the smallest length projectiles $(0.08 \mathrm{~m}$ long). Table 5 gives experimental values of final velocities and impact coefficients for $\alpha=45^{\circ}$ with impact velocities between 1.3 and 6.1 $\mathrm{m} / \mathrm{s}$ (corresponding to an impact energy range of 0.15-3.25 J). From these results, it is apparent that there is some tendency for the values of $\mu$ and $e$ to vary with the impact velocity, particularly in the latter case where $e$ changes from a mean of 0.407 at $1.3 \mathrm{~m} / \mathrm{s}$ to 0.273 at $4.2 \mathrm{~m} / \mathrm{s}$ (1.56 J impact energy). Over this range of velocity change, the mean value of $\mu$ decreases from 0.624 to 0.493 and there is evidence of more significant variation of $e_{m}$ than hitherto. In these tests insignificant longitudinal or transverse vibration was observed even at the higher impact velocities.

Finally, the influence of the test angle $\alpha$ on the impact coefficients was examined using the variable angle barrier (shown in Fig. 2) set at angles of $17^{\circ}, 25^{\circ}, 37^{\circ}$, and $60^{\circ}$. The results of tests carried out at these angles with similar impact energies are shown in Table 6 whilst corresponding results for an angle of $45^{\circ}$ are included in Table 5 (sequence "b"). A number of observations are pertinent: (i) the impulse ratio decreases as $\alpha$ decreases below $45^{\circ}$. (ii) $e_{m}$ shows considerable variation and changes to a mean of 0.47 at $\alpha=17^{\circ}$ from a value of -1.0 at $\alpha=60^{\circ}$. (iii) $e$ tends to decrease in a fairly consistent manner as $\alpha$ increases and (iv) as in all previous cases, the sum of measured energy losses for each angle closely relates to the initial impact energy.

\subsection{Discussion}

Before discussing individual aspects of the impact behaviour, two important general findings should be emphasised. It is first of all clear that even under the same experimental conditions there are substantial variations in (i) the measured velocities after 
impact and (ii) the resultant calculated impact coefficients. Moreover, within the range of these uncertainties which occur under similar test conditions there does not appear to be any correlation between any of the impact coefficients. An interesting implication is that a significant proportion of the variance in ignition probabilities determined from drop weight experiments [4] should be attributable to random effects in the mechanical impact process rather than to the combustion process itself. In the combustion process, initial heating up of the gases near the hot frictional surfaces creates a local volume element (ignition kernel) of hot gas that will continue (or not) to get hotter until ignition occurs. This is influenced by the diffusion and turbulence conditions of the gas mixture (subject to local variation) and to the surface temperature generated during impact and the duration of its influence [1]. Variation in the impact process and the dispersion of energy will manifest itself in changes to the generated surface temperature and thus the likelihood of ignition.

A second major finding is that it can be observed from the test results reported in Table 2, 4, 5 and 6, that in all cases the sum of tangential, normal and retained kinetic energy values after impact is closely similar to the initial impact energy. This clearly suggests that all of the energy losses that occur during impact are accounted for in the sum of the normal and tangential components, inferring that these include those from all sources: i.e. heat, vibration and any plastic deformation. Of particular interest in this context is the behaviour of the long $(0.24 \mathrm{~m})$ slender projectiles where significant vibrations and acoustic noise were observed during the experiments although all of the energy losses were still accounted for by normal and tangential effects. With eccentric impacts of the kind described in this paper, both compressive longitudinal (sound) and transverse waves will be 'generated in long projectiles. The first of these will result in acoustic noise whereas the latter will be manifested as bending of the projectile during and immediately after the impact: the result of these vibrations is to reduce the amount of the energy loss that can be associated with sliding friction, viscous friction or plastic deformation effects. In particular, the loss due to vibration must reduce the amount of the tangential energy loss that can be associated with frictional processes and so, in this respect might represent a significant error when considering the surface temperature increase during an impact.

Most of the energy of impact which is converted into vibration will eventually be transformed via a frictional process to manifest itself as heat in the bulk of the projectile, anvil/barrier and its surroundings (and thus will not significantly contribute to a temperature increase at the contact zone). Although it can be surmised that the amount of energy lost acoustically will be very small or even miniscule, further study carrying out sound measurements in a controlled acoustic environment would provide substantive evidence. The use of laser vibrometry or stress wave analysis techniques (SWAN) could also help in gaining an understanding of the role of vibration in determining energy losses in the impact process.

The insignificant vibration noted in the experiments using the smaller projectiles is in agreement with their lower "slenderness coefficients". According to Stoianovici and Hurmuzlu [16] who studied the rebound velocities of freely dropped bars onto an external surface, these slenderness coefficients indicate the applicability of rigid body impact dynamics for particular projectile shapes and sizes. Essentially, the slenderness coefficient factor identifies the class of collision problems where the coefficient of 
restitution is invariant to the inclination angle. For the steel bars employed by Stoianovici and Hurmuzlu, the slenderness coefficient $(\gamma)$ range which suggests applicability of rigid body theory is given by

$$
\gamma=\frac{l}{d_{a}{ }^{1.3815}} \leq 26.45 \mathrm{~m}^{-0.3815}
$$

So that $\gamma$ is approximately 57,29 and 19 respectively for the $0.24,0.12$ and $0.08 \mathrm{~m}$ long projectiles used in the present study. These are in accordance with the much more significant degree of vibration effects observed with the $0.24 \mathrm{~m}$ projectile and can be interpreted here in terms of the extent to which the normal and tangential energy losses include components arising from vibration

The degree to which fingermark surface contamination influences the impact coefficients is immediately obvious from the results given in Tables 2 and 3. A newly produced clean surface causes a reduction in $e$ suggesting that both elastic and plastic behaviour is influenced by surface contamination. Freshly machined impact surfaces resulted in much higher impulse values $\mu$ (typically more than twice as great as those for contaminated surfaces) which implies a clear association with frictional processes. This conclusion is further supported by the similarity of the impulse ratio values of the contaminated surfaces to sliding friction coefficient values measured previously [1]. Predictive calculation of the energy losses with Eq. 23-25, for conditions appertaining to Table 3 , shows that increasing $\mu$ from 0.32 to 0.72 will increase the proportion of the energy losses (w.r.t. impact energy) in accordance with the experimental observations (i.e. from $\approx 50 \%$ to $>90 \%$ ). This has clear implications concerning the generation of surface temperatures.

Cleaning the surfaces with an HFE based azeotrope (HD71E), whilst increasing the impulse ratio or friction coefficient, was not able to completely restore the surfaces to the pre-contamination state suggesting that tiny amounts of contamination, possibly of mono-molecular thickness were left on the surfaces. Whilst it is well known that such amounts of surface contamination can greatly affect sliding friction effects, the effect of plastic deformation during impact might suggest that new impact surfaces are created negating the influence of the original contamination. Since this is clearly not the case, it represents an important finding in the context of ignition testing indicating that the state of the impact surfaces and their handling need to be taken into account. The lack of significant change to measured impact velocities and impact coefficients when contaminated surfaces were subjected to several seconds of flaming is also of interest in relation to ignition testing: it can be taken that ignition test results in a sequence will not be influenced by preceding ignitions having occurred. The difficult question remains however, as to whether friction coefficient values relating to contaminated or uncontaminated surfaces should be used in practice for predicting energy losses where impacting surfaces will most likely be contaminated.

Examining the pattern of variation of the moment restitution coefficient $e_{m}$, it is apparent that the coefficient for the larger projectiles is consistently very close to a value of -1 corresponding to zero moment impulse with point forces at the contact. However, there is much more variation in the value of this coefficient with the smaller projectiles particularly with the low impact test angles. At $17^{\circ}$ impact angle (Table 6) the value of $e_{m}$ is significantly and consistently larger than for larger impact test angles and there seems 
to be a clear trend for it to decrease with the impact angle. In reality, impacting bodies cannot sustain non-zero forces at a single contact point and contact will occur over an area or region. Within the curved impact area of the projectile different points may be making contact with the anvil surface particularly if the projectile rolls, sticks (as is the case with low angle impacts), deforms or partially interlocks during the impact. In consequence it can be considered likely that the moment impulse would increase in value (n.b. Eq. 13) as $e_{m}$ increases with diminution of $\alpha$.

The experiments carried out with impacts at different anvil test angles reported in Table 6 showed that for angles of $37^{\circ}$ and lower, the tangential contact velocities changed direction (pre-impact contact velocity is equal to $v_{t}$ ) indicating that at some point sticking occurred with $V_{c t}=0$ before reversal of motion. Gillardi and Sharf [17] have given a useful description of graph impact analysis distinguishing between the ranges of impulse ratios associated with conditions of sticking and slipping followed by reverse motion. The latter condition is considered to arise [18] when sticking occurs before there is maximum compression during the approach impact phase. To find the critical impulse ratios corresponding to cessation of motion prior to separation, Eq. 10 was replaced by Eq. 11 as one of the system equations to determine $V_{t}$ and $V_{n}$ for the respective mean values of $v_{t}$ and $v_{n}$. The values of $\mu=\mu_{c}$, then obtained from Eq. 17, are shown plotted in Fig. 5 to reveal their relationship with the mean experimental impulse ratio values for different barrier inclination angles.

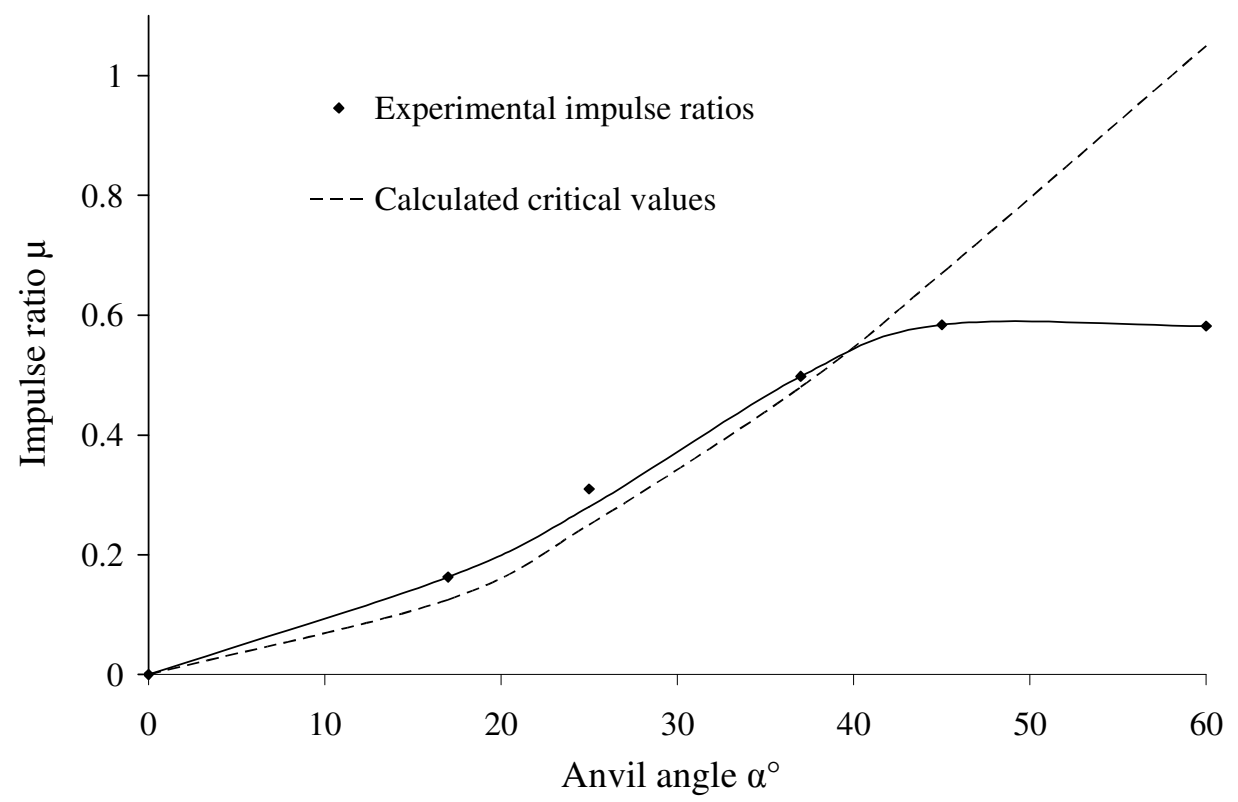

Figure 5. Relationship between the critical impulse ratio and mean experimental values for $0.08 \mathrm{~m}$ projectile with impact energy $0.43-0.47 \mathrm{~J}$.

The impulse ratio for angles where sticking was not observed, i.e. 45 and $60^{\circ}$, are similar in value to the sliding friction coefficient for these materials ${ }^{2}$ whereas at smaller angles the impulse ratio is in alignment with the critical impulse value. This clearly gives

\footnotetext{
${ }^{2}$ Friction coefficient values for steel on steel are widely available. E.g. $f=0.57$ Engineering Handbook. Com, accessed $28^{\text {th }}$ April 2016.
} 
support to (i) the applicability of the Amontons-Coulomb friction model to describe the tangential impact process and (ii) the contention of a limiting value of the impulse ratio for low impact test angles as proposed by Brach [7, 11, 12]. Brach defined a limiting value for impulse ratio such that under any condition the value of the tangential impulse can never be greater than $\mu_{c} P_{n}$. For impacts of a rigid body against a massive barrier, $\mu_{c}$ corresponds to the situation where sliding stops at separation.

It is interesting to explore the energy losses which occur during the low test angle impacts. From Table 6 it can be seen that, even though sticking has occurred with reverse motion, the sum of the energy losses together with the KE retained after impact is very close in value to the impact energy supporting the validity of the calculations. However, if values of impulse ratio greater than the critical value are used in any prediction of impact energy loss then unrealistic negative values of energy losses (i.e. with tangential energy gain) become possible. This is illustrated in Fig. 6 which shows the range of permissible positive energy losses (calculated with the corresponding experimental values of the restitution coefficients) for a range of impulse ratio values. For easier comparison the energy losses are shown in a non-dimensional form.

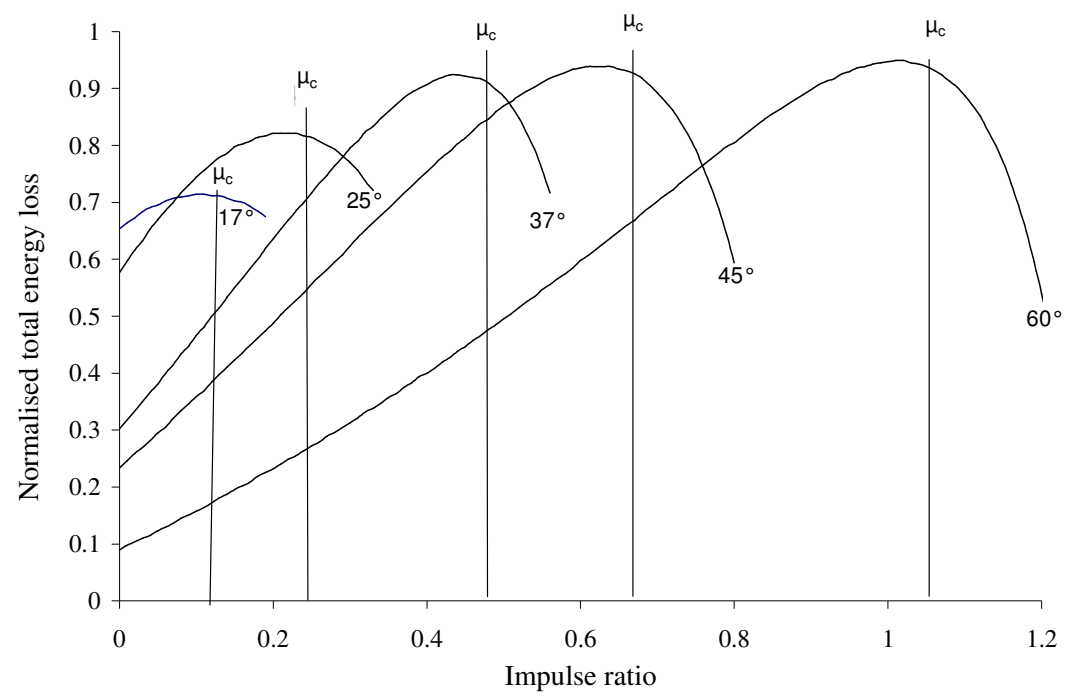

Figure 6. Prediction of total energy losses $\left(E_{l t}+E_{l n}\right)$ for the $0.08 \mathrm{~m}$ projectile impacting the variable angle anvil with restitution coefficients given in Table 5 (series b) and 6.

It is apparent that the critical impulse ratios are associated with total energy losses that are close to the maximum values. As Brach points out, this is a useful concept for many practical applications since it leads to prediction of maximum energy losses. This also has relevance to the development of safety case presentations in the nuclear industry since maximum energy losses would be associated with pessimistic probabilities of mechanical 
ignition.

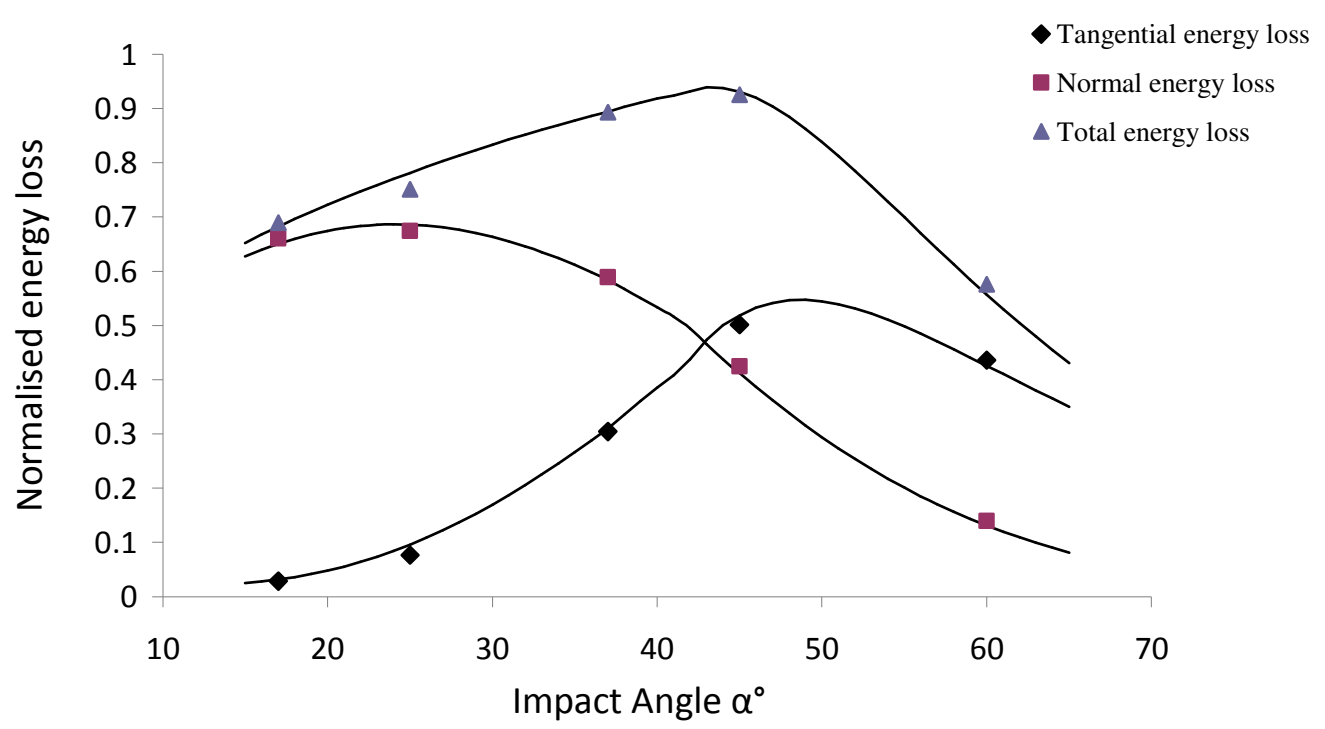

Figure 7. Normalised energy loss variation (0.08 m projectile) with impact angle. Markers show experimentally obtained values and line plots show calculated values (Eq. 23-25) using interpolated data.

The proportion of the total energy loss associated with sliding friction is of most interest from the perspective of this study. Normalised energy loss values shown in Fig. 7 suggest that (for these impact conditions) the maximum total energy losses occur at approximately $45^{\circ}$ whereas the maximum tangential energy loss appears to correspond to a slightly higher angular inclination $\left(\approx 50^{\circ}\right)$.

Finally, the usefulness of the impulse momentum approach must be considered in relation to its ability to provide a relatively straightforward means of determining energy loss values for the eventual estimation of surface temperature rises. It has been shown that tangential and normal energy losses values can be obtained that accurately account for the original impact energy. These energy losses may be determined from experimental values of the final velocities and angular motion (Eq. 20 and 21) or by employing Eq. 2325 with the initial velocities and apposite values of the impact coefficients $\left(e, e_{m}\right.$ and $\left.\mu\right)$. For practical purposes the issue is whether or not realistic values of the coefficients can be obtained for use with these expressions. For many impacts, the selection of a value for the coefficient of moment restitution may be relatively uncomplicated without the possibility of too much error being introduced. The value of the impulse ratio employed should not exceed the critical value and must take into account the condition of the impact surfaces which mostly determines the friction coefficient. From the results presented in this study it is apparent that the most important influence on the magnitude of the impulse ratio is contamination of the impact surfaces. It is widely recognized that the coefficient of restitution can show significant variation in value due to the influence of material properties, projectile shape, initial velocities and whether multiple collisions occur during impact [16]. Such variation is apparent from the experimental results given in Table 2 to 6 . In Table 5, for example, it can be seen that the value of $e$ tends to reduce 
as the initial impact velocity increases, representing typical behaviour for most rigid impact scenarios.

There are clearly difficulties associated with selection of appropriate values of impact coefficients and in some cases it might be necessary to consider arbitrary subintervals of time within the contact duration as described earlier (n.b. Eq. 22). To more fully understand the kinematic behaviour of sticking and reversal of the contact tip motion would require a faster video imaging system than employed in this study. However for many situations, unidirectional sliding can be assumed and the approach detailed here be considered to offer a relatively straightforward and useful means of assessing impact energy losses.

\subsection{Conclusions}

Full experimental details with measured velocity components have been provided for a test programme of impacting projectiles onto a massive barrier. Of major importance in the context of ignition testing involving mechanical stimuli, it was found that (i) there was substantial variation in the measured velocities after impact under closely similar test conditions and (ii) the presence of surface contamination on the impacting surfaces (resulting from exposure to the laboratory atmosphere and light manual handling) was sufficient to more than halve the measured values of the impulse ratio. It has been further shown that in all the tests carried out (even with long projectiles that exhibit very significant longitudinal and transverse vibration following impact) the entire energy loss can be accounted for in terms of normal and tangential components. Useful expressions have been derived to enable these components to be determined given knowledge of the initial velocities and the three impact coefficients. It is concluded that the experimentally determined energy losses include the effects of vibration so that the fraction of the tangential energy attributable to friction effects (which would result in the generation of a surface temperature increase) would be reduced according to the extent of the vibrations. It was determined from the high speed digital imaging, however, that such vibrations were insignificant with the shorter projectiles employed in the majority of the experiments.

The experimental data obtained in this work supports the approach taken by Brach [7, 11, 12] in several respects: (i) The concept of contact moment impulse appears to be appropriate to the experimental impacts with realistic values of the coefficient $e_{m}$ being recorded. In many cases, the value of $e_{m}$ was found to be close to -1 , indicating zero moment impulse and that the planar forces are represented as point forces at a given point. (ii) The results of experimental tests conducted with varying barrier inclination angles gives clear support to the applicability of the Amontons-Coulomb friction model to describe the tangential impact process and Brach`s postulation of a limiting critical impulse ratio at low inclination angles. These experimental results are also of interest in showing that under these impact conditions the maximum total energy losses occur with barrier angles at roughly $45^{\circ}$ whereas the maximum tangential loss (which would relate to maximum surface temperature increase due to sliding friction) corresponds to a slightly higher angular inclination.

In spite of the difficulties associated with selection of appropriate values of impact coefficients, it is finally concluded that given the great complexity of impact 
phenomena with its many variables and uncertainties, the moment impulse approach offers a relatively straightforward and useful means of assessing impact energy losses. Determination of energy losses in this manner should offer a future means of assessing likely surface temperature increases resulting from impacts of the kind which could cause ignition of flammable atmospheres.

\section{Acknowledgments}

The Hydrogen Hazards Unit at London South Bank University would like to acknowledge the financial support of Sellafield Ltd. and the University in undertaking this programme of work. Dr. Anthony Averill wishes to express his immense gratitude to Mr. Eric Chemla, Drs. Andrew Clarke and Daniela Mendela, and the Cardiovascular team at St. George`s NHS hospital, London, who saved his life following a ruptured aortic abdominal aneurysm in February 2016.

\section{References}

1. Averill AF, Ingram JM, Holborn PG, Battersby P, Nolan PF. Fundamental study of generation of interfacial temperatures with metal surfaces and coatings under conditions of sliding friction and mechanical impact: part 2: experimental determination of surface temperature. Trans. IMF 2014;92(2):99-107.

2. Averill AF, Ingram JM, Battersby P, Holborn PG. Ignition of flammable hydrogen/air mixtures by mechanical stimuli. Part 1: ignition with clean metal surfaces sliding under high load conditions. International Journal of Hydrogen Energy (2014); 39:18472-18479.

3. Averill AF, Ingram JM, Battersby P, Holborn PG. Ignition of flammable hydrogen/air mixtures by mechanical stimuli. Part 2: Ignition under conditions of rust and surface pyrophoric material contamination. International Journal of Hydrogen Energy 2015; 40:4392-4400.

4. Jones SJ, Averill AF, Ingram JM, Holborn PG, Battersby P, Nolan PF, et al. Impact ignition of hydrogen - air mixtures, In: Hazards XIX. Process Safety and Environmental Protection. Symposium Series No 151; IChemE; 2006. pp 401-409.

5. Averill AF, Ingram JM, Holborn PG, Battersby P, Nolan PF. Ignition of flammable hydrogen/air mixtures by controlled glancing impacts in nuclear decommissioning. Paper No 234, $5^{\text {th }}$ Int.Conf. on Hydrogen Safety, Sept. 9 - 11, 2013, Brussels, Belgium.

6. Averill AF, Ingram JM, Holborn PG, Battersby P, Nolan PF. Ignition of hydrogen/air mixtures by glancing mechanical impact. International Journal of Hydrogen Energy 2014;39:20404-20410.

7. Brach RM, Mechanical Impact Dynamics: Rigid Body Collisions. John Wiley and Sons. New York , 260 pp, 1991. 
8. Painlevé P. Sur le lois frottement de glissemment. C.R. Acad Sci (Paris) 1895;121:112115.

9. Champneys AR and Varkoni PL. The Painlevé paradox in contact mechanics. arXiv:1601.03545v1[physics.class-ph] 14 Jan 2016.

10. Zhao Z, Liu C, Chen B. The Painlevé paradox studied at a 3D slender rod. Multibody Syst Dyn 2007; 19(4):323-43.

11. Brach R M, Impact dynamics with applications to solid particle erosion. International Journal of Impact Engineering 1988;7:37-53.

12. Brach RM, Classical planar impact theory and the tip impact of a slender rod. International Journal of Impact Engineering 1993;13(1):21-33.

13. Stronge WJ. Chap 3. Impact Mechanics. Camb. Univ. Press. 2004.

14. Averill AF, Ingram $J$ A and Nolan P.F. On the performance and mechanism of ultrasonically cleaning metal components with environmentally acceptable organic solvents. Trans. IMF 1999;77(6):230-236.

15. Girod A, Ramotowski R and Weyermann C. Composition of fingermark residue: A qualitative and quantitative review. Forensic Science International 2012;223:10-24.

16. Stoianovici SP and Hurmuzlu Y, A critical study of the applicability of rigid body collision theory, ASME Journal of Applied Mechanics, 1996;63(2):307-316.

17. Gilardi G, Sharf I. Literature survey of contact dynamics modelling. Mechanism and Machine Theory 2002;37:1213-1239.

18. Han I and Gilmore BJ. Multi-body impact motion with friction analysis, simulation and experimental validation. J. Mech Design 1993;115:412-422. 


\begin{tabular}{|c|c|c|c|c|c|c|c|c|c|c|c|c|c|c|}
\hline Test & $\mathbf{v}_{\mathbf{n}} \mathbf{m} / \mathbf{s}$ & $\mathbf{v}_{\mathrm{t}} \mathbf{m} / \mathbf{s}$ & $\mathbf{V}_{\mathbf{n}} \mathbf{m} / \mathbf{s}$ & $\mathrm{V}_{\mathrm{t}} \mathbf{m} / \mathbf{s}$ & $\Omega \mathrm{r} / \mathrm{s}$ & $\mathbf{V}_{\mathrm{cn}} \mathrm{m} / \mathrm{s}$ & $\mathbf{V}_{\text {ct }} \mathbf{m} / \mathbf{s}$ & $\mathbf{e}$ & $\mathbf{e}_{m}$ & $\mu$ & $\mathbf{E}_{\mathbf{l t}} \mathbf{J}$ & $\mathbf{E}_{\ln } \mathbf{J}$ & $\mathbf{E}_{\mathbf{r}} \mathbf{J}$ & $\mathbf{E}_{\text {total }} \mathbf{J}$ \\
\hline 1 & -2.751 & -2.809 & -0.397 & -1.887 & 26.94 & 2.14 & -4.05 & 0.678 & -1.10 & 0.392 & 1.616 & 0.537 & 1.809 & 3.962 \\
\hline 2 & -2.651 & -2.651 & -0.483 & -1.920 & 25.84 & 1.95 & -3.99 & 0.637 & -1.05 & 0.337 & 1.241 & 0.536 & 1.789 & 3.566 \\
\hline 3 & -2.790 & -2.782 & -0.375 & -1.984 & 28.36 & 2.26 & -4.30 & 0.719 & -1.03 & 0.331 & 1.437 & 0.490 & 1.993 & 3.920 \\
\hline 4 & -2.737 & -2.730 & -0.486 & -2.059 & 27.73 & 2.09 & -4.35 & 0.673 & -1.03 & 0.298 & 1.203 & 0.519 & 2.050 & 3.772 \\
\hline 5 & -2.725 & -2.742 & -0.411 & -2.047 & 29.32 & 2.31 & -4.45 & 0.753 & -1.06 & 0.300 & 1.270 & 0.406 & 2.131 & 3.807 \\
\hline 6 & -2.765 & -2.824 & -0.585 & -2.250 & 28.95 & 2.13 & -4.61 & 0.668 & -1.05 & 0.264 & 1.085 & 0.516 & 2.366 & 3.967 \\
\hline 7 & -2.785 & -2.783 & -0.484 & -2.084 & 28.94 & 2.23 & -4.44 & 0.699 & -1.06 & 0.304 & 1.285 & 0.499 & 2.159 & 3.943 \\
\hline 8 & -2.797 & -2.823 & -0.399 & -1.959 & 28.08 & 2.20 & -4.26 & 0.700 & -1.07 & 0.360 & 1.555 & 0.519 & 1.954 & 4.028 \\
\hline 9 & -2.787 & -2.776 & -0.728 & -2.437 & 29.90 & 2.09 & -4.87 & 0.640 & -1.01 & 0.165 & 0.660 & 0.532 & 2.700 & 3.892 \\
\hline 10 & -2.762 & -2.833 & -0.409 & -1.877 & 25.92 & 2.00 & -4.01 & 0.640 & -1.09 & 0.406 & 1.658 & 0.600 & 1.736 & 3.994 \\
\hline 11 & -2.801 & -2.813 & -0.445 & -1.956 & 26.48 & 2.00 & -4.16 & 0.635 & -1.04 & 0.364 & 1.510 & 0.617 & 1.855 & 3.982 \\
\hline 12 & -2.791 & -2.801 & -0.405 & -1.942 & 26.99 & 2.13 & -4.17 & 0.667 & -1.04 & 0.359 & 1.517 & 0.570 & 1.866 & 3.953 \\
\hline 13 & -2.789 & -2.783 & -0.425 & -1.969 & 27.27 & 2.13 & -4.20 & 0.668 & -1.03 & 0.344 & 1.444 & 0.562 & 1.915 & 3.921 \\
\hline 14 & -2.796 & -2.813 & -0.591 & -2.222 & 28.24 & 2.03 & -4.56 & 0.637 & -1.04 & 0.268 & 1.103 & 0.575 & 2.288 & 3.966 \\
\hline 15 & -2.825 & -2.832 & -0.544 & -2.128 & 28.19 & 2.09 & -4.45 & 0.645 & -1.05 & 0.309 & 1.300 & 0.587 & 2.169 & 4.056 \\
\hline 16 & -2.826 & -2.931 & -0.320 & -1.753 & 25.50 & 2.02 & -3.89 & 0.644 & -1.10 & 0.430 & 2.025 & 0.645 & 1.581 & 4.251 \\
\hline 17 & -2.845 & -2.831 & -0.403 & -1.952 & 26.09 & 2.01 & -4.13 & 0.629 & -0.98 & 0.360 & 1.544 & 0.661 & 1.818 & 4.023 \\
\hline 18 & -2.812 & -2.834 & -0.399 & -1.917 & 27.61 & 2.19 & -4.18 & 0.683 & -1.08 & 0.380 & 1.634 & 0.555 & 1.882 & 4.071 \\
\hline 19 & -2.832 & -2.845 & -0.601 & -2.358 & 30.78 & 2.30 & -4.85 & 0.700 & -1.03 & 0.219 & 0.956 & 0.490 & 2.628 & 4.074 \\
\hline 20 & -2.780 & -2.839 & -0.287 & -1.787 & 25.42 & 2.09 & -3.88 & 0.665 & -1.04 & 0.422 & 1.792 & 0.597 & 1.602 & 3.991 \\
\hline 21 & -2.795 & -2.862 & -0.463 & -2.030 & 27.23 & 2.11 & -4.23 & 0.653 & -1.06 & 0.357 & 1.505 & 0.582 & 1.982 & 4.069 \\
\hline 22 & -2.857 & -2.860 & -0.198 & -1.673 & 26.75 & 2.32 & -3.86 & 0.717 & -1.07 & 0.446 & 2.028 & 0.555 & 1.576 & 4.159 \\
\hline 23 & -2.826 & -2.842 & -0.509 & -2.153 & 29.78 & 2.29 & -4.59 & 0.705 & -1.07 & 0.297 & 1.302 & 0.500 & 2.298 & 4.100 \\
\hline 24 & -2.803 & -2.844 & -0.704 & -2.391 & 29.38 & 2.01 & -4.82 & 0.629 & -1.04 & 0.216 & 0.879 & 0.561 & 2.599 & 4.039 \\
\hline 25 & -2.782 & -2.852 & -0.614 & -2.278 & 30.05 & 2.16 & -4.78 & 0.665 & -1.10 & 0.254 & 1.107 & 0.489 & 2.485 & 4.081 \\
\hline 26 & -2.808 & -2.838 & -0.360 & -1.904 & 26.93 & 2.15 & -4.12 & 0.677 & -1.04 & 0.382 & 1.648 & 0.571 & 1.817 & 4.036 \\
\hline 27 & -2.747 & -2.782 & -0.394 & -1.921 & 25.77 & 2.00 & -4.05 & 0.645 & -1.01 & 0.366 & 1.489 & 0.589 & 1.766 & 3.844 \\
\hline
\end{tabular}




\begin{tabular}{|c|c|c|c|c|c|c|c|c|c|c|c|c|c|c|}
\hline 38 & -2.854 & -2.848 & -0.316 & -1.918 & 27.15 & 2.21 & -4.16 & 0.688 & -0.99 & 0.366 & 1.651 & 0.582 & 1.838 & 4.071 \\
\hline 29 & -2.815 & -2.834 & -0.354 & -1.894 & 26.37 & 2.12 & -4.07 & 0.661 & -1.02 & 0.382 & 1.644 & 0.603 & 1.770 & 4.017 \\
\hline 30 & -2.821 & -2.824 & -0.284 & -1.864 & 27.83 & 2.31 & -4.16 & 0.728 & -1.04 & 0.379 & 1.700 & 0.504 & 1.827 & 4.031 \\
\hline Mean & -2.792 & -2.815 & -0.446 & -2.017 & 27.66 & 2.14 & -4.29 & 0.672 & -1.05 & 0.335 & 1.426 & 0.552 & 2.008 & 3.986 \\
\hline SD & 0.042 & 0.049 & 0.123 & 0.188 & 1.48 & 0.11 & 0.29 & 0.032 & 0.03 & 0.067 & 0.314 & 0.053 & 0.311 & 0.126 \\
\hline
\end{tabular}

Table 3. Effect of tip and anvil test plate condition on the impact coefficients. $0.24 \mathrm{~m}$ steel projectile (tip profile B) dropped onto a massive steel barrier. $\alpha=\pi / 4\left(45^{\circ}\right) . v_{n} \approx$ $1.6 \mathrm{~m} / \mathrm{s}$ and $v_{t} \approx 1.6 \mathrm{~m} / \mathrm{s} . d_{c}=0.083 \mathrm{~m}$ and $d_{d}=0.086 \mathrm{~m}$. Mean impact energy 1.3

Joules. Mean and standard deviation values shown respectively in the bottom two rows.

\begin{tabular}{|c|c|c|c|c|c|c|c|c|}
\hline \multicolumn{3}{|c|}{ Freshly machined tip } & \multicolumn{3}{|c|}{ Contaminated tip } & \multicolumn{3}{|c|}{ Cleaned with HFE 71DE* } \\
\hline $\mathrm{e}$ & $\mathrm{e}_{\mathrm{m}}$ & $\mu$ & $\mathrm{e}$ & $e_{m}$ & $\mu$ & $\mathrm{e}$ & $e_{m}$ & $\mu$ \\
\hline 0.543 & -1.130 & 0.741 & 0.674 & -1.020 & 0.331 & 0.636 & -0.979 & 0.587 \\
\hline 0.573 & $-0,929$ & 0.737 & 0.629 & -0.949 & 0.354 & 0.580 & -1.013 & 0.482 \\
\hline 0.607 & -1.005 & 0.750 & 0.718 & -1.051 & 0.358 & 0.561 & -0.995 & 0.616 \\
\hline 0.580 & -0.958 & 0.699 & 0.680 & -1.037 & 0.267 & 0.640 & -1.009 & 0.474 \\
\hline 0.546 & -0.971 & 0.728 & 0.630 & -1.037 & 0.247 & 0.605 & -1.062 & 0.623 \\
\hline 0.568 & -1.132 & 0.801 & 0.672 & -1.020 & 0.391 & 0.572 & -0.980 & 0.596 \\
\hline 0.545 & -1.043 & 0.735 & 0.684 & -1.029 & 0.391 & 0.522 & -1.022 & 0.619 \\
\hline 0.508 & -0.969 & 0.726 & 0.67 & -0.984 & 0.262 & 0.566 & -0.998 & 0.47 \\
\hline 0.597 & -1.055 & 0.687 & 0.604 & -0.985 & 0.327 & 0.579 & -0.946 & 0.654 \\
\hline 0.536 & -0.911 & 0.681 & 0.687 & -1.029 & 0.356 & 0.661 & -1.012 & 0.533 \\
\hline 0.508 & -0.902 & 0.678 & 0.74 & -1.074 & 0.172 & 0.587 & -1.1352 & 0.673 \\
\hline 0.560 & -0.942 & 0.720 & 0.641 & -0.892 & 0.380 & 0.581 & -1.006 & 0.590 \\
\hline 0.556 & -0.996 & 0.724 & 0.669 & -1.0145 & 0.320 & \begin{tabular}{|l|}
0.591 \\
\end{tabular} & -1.013 & 0.577 \\
\hline 0.031 & 0.079 & 0.035 & 0.038 & 0.038 & 0.068 & 0.388 & 0.475 & 0.070 \\
\hline
\end{tabular}

* Tip and anvil test plate were wipe-cleaned for the first 4 tests. Ultrasonic cleaning was employed for the remaining tests. 
Table 4. Experimentally determined velocities, impact coefficients and kinetic energies for tests with $0.12 \mathrm{~m}$ long steel projectile (tip profile B) dropped onto a massive steel barrier. $\alpha=\pi / 4\left(45^{\circ}\right) . d_{c}=0.0364 \mathrm{~m}$ and $d_{d}=0.048 \mathrm{~m}$. Mean impact velocity $4.2 \mathrm{~m} / \mathrm{s}$. Mean impact energy 2.28 J. Freshly machined impact surfaces.

\begin{tabular}{|c|c|c|c|c|c|c|c|c|c|c|c|c|c|c|}
\hline Test & $\mathbf{v}_{\mathrm{n}} \mathrm{m} / \mathrm{s}$ & $v_{t} \mathbf{m} / s$ & $\mathbf{V}_{\mathrm{n}} \mathrm{m} / \mathrm{s}$ & $\mathrm{V}_{\mathrm{t}} \mathrm{m} / \mathrm{s}$ & $\Omega \mathrm{r} / \mathrm{s}$ & $\mathrm{V}_{\mathrm{cn}} \mathrm{m} / \mathrm{s}$ & $\mathrm{V}_{\mathrm{ct}} \mathrm{m} / \mathrm{s}$ & $\mathbf{e}$ & $\mathbf{e}_{\mathrm{m}}$ & $\mu$ & $E_{l t} J$ & $\mathbf{E}_{\ln } \mathbf{J}$ & $\mathbf{E}_{\mathbf{r}} \mathbf{J}$ & $\mathbf{E}_{\text {total }} \mathbf{J}$ \\
\hline 1 & -3.012 & -2.910 & -0.265 & -1.587 & 28.03 & 0.82 & -2.53 & 0.247 & -0.95 & 0.482 & 1.013 & 0.813 & 0.465 & 2.291 \\
\hline 2 & -3.028 & -3.008 & -0.142 & -1.517 & 30.03 & 1.04 & -2.51 & 0.310 & -1.11 & 0.517 & 1.166 & 0.786 & 0.449 & 2.401 \\
\hline 3 & -2.963 & -2.911 & -0.357 & -1.488 & 25.82 & 0.68 & -2.34 & 0.193 & -1.21 & 0.546 & 1.052 & 0.813 & 0.414 & 2.279 \\
\hline 4 & -2.956 & -2.925 & -0.090 & -1.330 & 21.87 & 0.77 & -2.10 & 0.236 & -0.98 & 0.557 & 1.109 & 0.845 & 0.310 & 2.264 \\
\hline 5 & -2.948 & -2.915 & -0.185 & -1.576 & 25.86 & 0.85 & -2.45 & 0.253 & -0.88 & 0.484 & 1.006 & 0.794 & 0.438 & 2.238 \\
\hline 6 & -3.006 & -2.900 & 0.010 & -1.067 & 15.35 & 0.65 & -1.55 & 0.187 & -0.87 & 0.608 & 1.13 & 0.964 & 0.188 & 2.282 \\
\hline 7 & -2.995 & -2.940 & -0.726 & -1.768 & 21.71 & 0.05 & -2.56 & 0.018 & -1.02 & 0.517 & 0.883 & 0.872 & 0.555 & 2.310 \\
\hline 8 & -2.946 & -2.867 & 0.031 & -1.353 & 21.05 & 0.79 & -2.10 & 0.268 & -0.73 & 0.509 & 1.038 & 0.838 & 0.311 & 2.187 \\
\hline Mean & -2.982 & -2.922 & -0.215 & -1.461 & 23.71 & 0.71 & -2.27 & 0.214 & -0.97 & 0.527 & 1.050 & 0.841 & 0.391 & 2.282 \\
\hline SD & 0.032 & 0.041 & 0.244 & 0.211 & 4.66 & 0.29 & 0.34 & 0.088 & 0.15 & 0.042 & 0.088 & $\mathbf{0 . 0 5 7}$ & 0.115 & 0.061 \\
\hline
\end{tabular}

Table 5. Experimentally determined velocities, impact coefficients and kinetic energies for tests with $0.08 \mathrm{~m}$ long steel projectile (tip profile B) dropped onto a massive steel barrier at different velocities. $\alpha=\pi / 4\left(45^{\circ}\right) . d_{c}=0.0226 \mathrm{~m}$ and $d_{d}=0.035 \mathrm{~m}$. Mean impact energies (a) 0.15 , (b) 0.39 , (c) 1.56 and (d) $3.25 \mathrm{~J}$. Freshly machined impact surfaces.

\begin{tabular}{|c|c|c|c|c|c|c|c|c|c|c|c|c|c|c|}
\hline Test & $\mathbf{v}_{\mathrm{n}} \mathrm{m} / \mathrm{s}$ & $\mathbf{v}_{\mathrm{t}} \mathrm{m} / \mathrm{s}$ & $\mathbf{V}_{\mathrm{n}} \mathrm{m} / \mathrm{s}$ & $V_{t} \mathrm{~m} / \mathrm{s}$ & $\Omega \mathrm{r} / \mathrm{s}$ & $\mathrm{V}_{\mathrm{cn}} \mathrm{m} / \mathrm{s}$ & $\mathrm{V}_{\mathrm{ct}} \mathrm{m} / \mathrm{s}$ & $\mathbf{e}$ & $\mathbf{e}_{m}$ & $\mu$ & $\mathbf{E}_{\mathrm{lt}} \mathbf{J}$ & $\mathbf{E}_{\ln } \mathbf{J}$ & $\mathbf{E}_{\mathbf{r}} \mathbf{J}$ & $\mathbf{E}_{\text {total }} \mathbf{J}$ \\
\hline $1 \mathrm{a}$ & -0.962 & -0.933 & 0.321 & -0.133 & 1.440 & 0.348 & -0.173 & 0.368 & -0.80 & 0.624 & 0.078 & 0.068 & 0.011 & 0.157 \\
\hline $2 a$ & -0.952 & -0.926 & 0.383 & -0.073 & 0.459 & 0.399 & -0.078 & 0.414 & -0.80 & 0.639 & 0.076 & 0.065 & 0.013 & 0.154 \\
\hline $3 a$ & -0.936 & -0.884 & 0.336 & -0.157 & 1.716 & 0.409 & -0.162 & 0.401 & -0.29 & 0.572 & 0.070 & 0.062 & 0.012 & 0.144 \\
\hline $4 \mathrm{a}$ & -0.922 & -0.926 & 0.397 & -0.121 & 3.124 & 0.448 & -0.213 & 0.507 & -1.07 & 0.611 & 0.081 & 0.052 & 0.016 & 0.149 \\
\hline $5 \mathrm{a}$ & -0.931 & -0.910 & 0.326 & -0.144 & 2.886 & 0.370 & -0.226 & 0.420 & -1.01 & 0.610 & 0.077 & 0.059 & 0.011 & 0.147 \\
\hline $6 a$ & -0.930 & -0.918 & 0.348 & -0.111 & 1.957 & 0.290 & -0.266 & 0.422 & -1.66 & 0.631 & 0.077 & 0.060 & 0.012 & 0.149 \\
\hline $7 \mathrm{a}$ & -0.944 & -0.913 & 0.310 & -0.149 & 0.845 & 0.324 & -0.173 & 0.349 & -0.29 & 0.609 & 0.073 & 0.067 & 0.010 & 0.150 \\
\hline $8 \mathrm{a}$ & -0.916 & -0.884 & 0.318 & -0.125 & 1.090 & 0.331 & -0.161 & 0.375 & -0.45 & 0.615 & 0.070 & 0.062 & 0.010 & 0.142 \\
\hline Mean & -0.937 & -0.912 & 0.343 & -0.127 & 1.69 & 0.365 & -0.181 & 0.407 & -0.80 & 0.614 & 0.075 & 0.062 & 0.012 & 0.149 \\
\hline SD & 0.015 & 0.018 & 0.032 & 0.027 & 0.94 & 0.052 & 0.056 & 0.048 & 0.46 & 0.020 & 0.004 & 0.005 & 0.002 & 0.005 \\
\hline
\end{tabular}




\begin{tabular}{|c|c|c|c|c|c|c|c|c|c|c|c|c|c|c|}
\hline $1 \mathrm{~b}$ & -1.572 & -1.602 & 0.381 & -0.371 & 3.607 & 0.458 & -0.457 & 0.294 & -1.94 & 0.631 & 0.226 & 0.189 & 0.025 & 0.440 \\
\hline $2 \mathrm{~b}$ & -1.516 & -1.507 & 0.271 & -0.369 & 1.019 & 0.261 & -0.429 & 0.194 & -1.01 & 0.637 & 0.190 & 0.191 & 0.018 & 0.399 \\
\hline $3 \mathrm{~b}$ & -1.475 & -1.475 & 0.340 & -0.338 & 2.933 & 0.334 & -0.472 & 0.275 & -1.36 & 0.627 & 0.191 & 0.170 & 0.020 & 0.381 \\
\hline $4 \mathrm{~b}$ & -1.477 & -1.507 & 0.382 & -0.368 & 6.586 & 0.528 & -0.522 & 0.359 & -1.71 & 0.613 & 0.210 & 0.154 & 0.027 & 0.391 \\
\hline $6 \mathrm{~b}$ & -1.487 & -1.391 & 0.364 & -0.428 & 5.202 & 0.500 & -0.528 & 0.324 & -0.36 & 0.520 & 0.169 & 0.163 & 0.029 & 0.361 \\
\hline $7 \mathrm{~b}$ & -1.539 & -1.497 & 0.531 & -0.258 & 3.096 & 0.577 & -0.351 & 0.390 & -0.50 & 0.599 & 0.202 & 0.170 & 0.031 & 0.403 \\
\hline $8 \mathrm{~b}$ & -1.496 & -1.454 & 0.349 & -0.556 & 14.592 & 0.682 & -0.868 & 0.454 & -0.79 & 0.487 & 0.198 & 0.132 & 0.048 & 0.378 \\
\hline SD & 0.038 & 0.064 & 0.073 & 0.085 & 4.139 & 0.134 & 0.155 & 0.078 & 0.60 & 0.056 & 0.018 & 0.019 & 0.009 & 0.026 \\
\hline $1 \mathrm{c}$ & -2.981 & -2.972 & 0.297 & -1.089 & 15.742 & 0.691 & -1.405 & 0.218 & -1.12 & 0.574 & 0.760 & 0.669 & 0.124 & 1.553 \\
\hline $2 \mathrm{c}$ & -2.998 & -2.982 & 0.189 & -1.231 & 22.565 & 0.755 & -1.688 & 0.233 & -1.21 & 0.549 & 0.767 & 0.642 & 0.161 & 1.570 \\
\hline $3 \mathrm{c}$ & -3.018 & -3.016 & 0.175 & -1.280 & 27.749 & 0.894 & -1.816 & 0.266 & -1.36 & 0.544 & 0.800 & 0.619 & 0.184 & 1.603 \\
\hline $4 \mathrm{c}$ & -2.966 & -2.922 & 0.062 & -1.683 & 41.734 & 1.105 & -2.562 & 0.339 & -0.93 & 0.409 & 0.657 & 0.519 & 0.333 & 1.510 \\
\hline $5 \mathrm{c}$ & -3.077 & -2.957 & -0.110 & -1.386 & 26.400 & 0.570 & -1.921 & 0.158 & -1.22 & 0.530 & 0.724 & 0.672 & 0.203 & 1.600 \\
\hline $6 \mathrm{c}$ & -2.999 & -2.945 & 0.015 & -1.726 & 42.364 & 1.090 & -2.567 & 0.324 & -0.93 & 0.404 & 0.656 & 0.534 & 0.348 & 1.539 \\
\hline $7 \mathrm{c}$ & -3.000 & -2.981 & 0.162 & -1.509 & 33.567 & 1.013 & -2.206 & 0.307 & -0.94 & 0.465 & 0.729 & 0.575 & 0.256 & 1.562 \\
\hline Mean & -3.012 & -2.970 & 0.123 & -1.420 & 30.925 & 0.909 & -2.049 & 0.273 & -1.09 & 0.493 & 0.733 & 0.601 & 0.233 & 1.567 \\
\hline SD & 0.038 & 0.029 & 0.127 & 0.220 & 9.456 & 0.216 & 0.415 & 0.066 & 0.16 & 0.065 & 0.053 & 0.059 & 0.080 & 0.033 \\
\hline $1 \mathrm{~d}$ & -4.281 & -4.274 & 0.479 & -1.610 & 37.977 & 1.458 & -2.334 & 0.312 & -1.47 & 0.560 & 1.682 & 1.226 & 0.317 & 3.225 \\
\hline $2 d$ & -4.300 & -4.239 & 0.111 & -1.910 & 46.514 & 1.258 & -2.862 & 0.270 & -1.42 & 0.528 & 1.585 & 1.211 & 0.426 & 3.222 \\
\hline $3 \mathrm{~d}$ & -4.389 & -4.264 & 1.191 & -1.095 & 22.765 & 1.702 & -1.626 & 0.389 & -0.83 & 0.568 & 1.707 & 1.310 & 0.254 & 3.271 \\
\hline $4 \mathrm{~d}$ & -4.370 & -4.280 & 0.232 & -2.068 & 55.342 & 1.702 & -3.058 & 0.339 & -1.16 & 0.481 & 1.603 & 1.163 & 0.528 & 3.294 \\
\hline $5 \mathrm{~d}$ & -4.317 & -4.264 & 0.174 & -1.717 & 45.008 & 1.370 & -2.545 & 0.276 & -2.03 & 0.567 & 1.684 & 1.229 & 0.359 & 3.272 \\
\hline $6 \mathrm{~d}$ & -4.358 & -4.362 & 0.388 & -2.077 & 57.729 & 1.784 & -3.188 & 0.389 & -1.18 & 0.481 & 1.691 & 1.106 & 0.553 & 3.350 \\
\hline $7 \mathrm{~d}$ & -4.318 & -4.297 & 0.515 & -1.674 & 33.171 & 1.318 & -2.366 & 0.293 & -1.06 & 0.543 & 1.637 & 1.291 & 0.322 & 3.250 \\
\hline $8 \mathrm{~d}$ & -4.305 & -4.306 & 0.412 & -1.970 & 64.552 & 2.030 & -3.120 & 0.435 & -1.45 & 0.495 & 1.745 & 1.004 & 0.557 & 3.306 \\
\hline Mean & -4.330 & -4.286 & 0.438 & -1.765 & 45.382 & 1.578 & -2.637 & 0.338 & -1.32 & 0.528 & 1.667 & 1.193 & 0.414 & 3.274 \\
\hline SD & 0.038 & 0.037 & 0.337 & 0.324 & 13.815 & 0.269 & 0.529 & 0.060 & 0.36 & 0.037 & 0.054 & 0.100 & 0.119 & 0.043 \\
\hline
\end{tabular}




\begin{tabular}{|c|c|c|c|c|c|c|c|c|c|c|c|c|c|c|}
\hline Test & $\mathbf{v}_{\mathbf{n}} \mathbf{m} / \mathbf{s}$ & $\mathbf{v}_{\mathrm{t}} \mathbf{m} / \mathbf{s}$ & $\mathbf{V}_{\mathrm{n}} \mathbf{m} / \mathbf{s}$ & $\mathbf{V}_{\mathrm{t}} \mathbf{m} / \mathbf{s}$ & $\mathbf{\Omega} \mathbf{r} / \mathbf{s}$ & $\mathbf{V}_{\mathrm{cn}} \mathbf{m} / \mathbf{s}$ & $\mathbf{V}_{\text {ct }} \mathbf{m} / \mathbf{s}$ & $\mathbf{e}$ & $\mathbf{e}_{m}$ & $\boldsymbol{\mu}$ & $\mathbf{E}_{\mathrm{lt}} \mathbf{J}$ & $\mathbf{E}_{\ln } \mathbf{J}$ & $\mathbf{E}_{\mathbf{r}} \mathbf{J}$ & $\mathbf{E}_{\text {total }} \mathbf{J}$ \\
\hline \multicolumn{15}{|c|}{$\alpha=17^{\circ} .0 .944 \pi \mathrm{rad}$. Mean impact energy $0.43 \mathrm{~J}$} \\
\hline 1 & -2.142 & -0.655 & 1.178 & 0.000 & -12.718 & 1.128 & 0.397 & 0.521 & -0.42 & 0.197 & 0.008 & 0.291 & 0.129 & 0.428 \\
\hline 2 & -2.137 & -0.622 & 1.247 & 0.005 & -14.623 & 1.092 & 0.442 & 0.549 & -0.52 & 0.185 & 0.001 & 0.277 & 0.146 & 0.424 \\
\hline 3 & -2.123 & -0.630 & 1.148 & -0.095 & -10.398 & 1.027 & 0.214 & 0.516 & -0.48 & 0.164 & 0.014 & 0.288 & 0.121 & 0.423 \\
\hline 4 & -2.141 & -0.640 & 1.198 & -0.050 & -11.215 & 1.127 & 0.303 & 0.533 & -0.44 & 0.177 & 0.012 & 0.285 & 0.132 & 0.429 \\
\hline 5 & -2.156 & -0.636 & 1.227 & -0.062 & -9.374 & 1.164 & 0.235 & 0.547 & -0.39 & 0.170 & 0.016 & 0.283 & 0.136 & 0.435 \\
\hline 6 & -2.121 & -0.621 & 1.192 & -0.103 & -9.280 & 1.174 & 0.204 & 0.540 & -0.46 & 0.156 & 0.016 & 0.277 & 0.129 & 0.422 \\
\hline 7 & -2.130 & -0.625 & 1.165 & -0.086 & -10.624 & 1.035 & 0.223 & 0.522 & -0.48 & 0.164 & 0.013 & 0.287 & 0.125 & 0.425 \\
\hline 8 & -2.112 & -0.620 & 1.058 & -0.126 & -9.828 & 0.969 & 0.178 & 0.478 & -0.51 & 0.156 & 0.015 & 0.301 & 0.104 & 0.420 \\
\hline Mean & -2.131 & -0.628 & 1.177 & -0.074 & -10.763 & 1.084 & 0.257 & 0.527 & -0.47 & 0.167 & 0.013 & 0.286 & 0.127 & 0.426 \\
\hline SD & 0.014 & 0.012 & 0.058 & 0.048 & 1.836 & 0.073 & 0.097 & 0.023 & 0.05 & 0.015 & 0.005 & 0.008 & 0.012 & 0.005 \\
\hline \multicolumn{15}{|c|}{$\alpha=25^{\circ} .0 .139 \pi \mathrm{rad}$. Mean impact energy $0.43 \mathrm{~J}$} \\
\hline 1 & -2.067 & -0.917 & 1.037 & -0.006 & -13.037 & 0.855 & 0.358 & 0.441 & -1.13 & 0.294 & 0.032 & 0.314 & 0.102 & 0.448 \\
\hline 2 & -2.044 & -0.939 & 1.177 & 0.054 & -10.259 & 1.063 & 0.359 & 0.527 & -0.66 & 0.308 & 0.041 & 0.272 & 0.127 & 0.440 \\
\hline 3 & -2.021 & -0.887 & 1.038 & 0.023 & -12.167 & 0.917 & 0.391 & 0.456 & -0.99 & 0.297 & 0.030 & 0.294 & 0.102 & 0.426 \\
\hline 4 & -1.983 & -0.916 & 1.070 & 0.024 & -9.863 & 1.040 & 0.360 & 0.491 & -0.68 & 0.308 & 0.041 & 0.270 & 0.105 & 0.416 \\
\hline 5 & -2.010 & -0.885 & 1.133 & 0.063 & -12.662 & 0.962 & 0.425 & 0.503 & -0.93 & 0.302 & 0.026 & 0.275 & 0.121 & 0.422 \\
\hline 6 & -2.044 & -0.936 & 1.117 & 0.052 & -12.005 & 1.030 & 0.434 & 0.490 & -0.75 & 0.312 & 0.035 & 0.289 & 0.116 & 0.440 \\
\hline 7 & -2.015 & -0.906 & 0.961 & 0.044 & -12.427 & 0.778 & 0.406 & 0.417 & -0.75 & 0.319 & 0.030 & 0.306 & 0.088 & 0.424 \\
\hline 8 & -2.028 & -0.936 & 0.940 & 0.078 & -13.099 & 0.812 & 0.470 & 0.401 & -0.61 & 0.342 & 0.030 & 0.316 & 0.086 & 0.432 \\
\hline Mean & -2.027 & -0.915 & 1.059 & 0.042 & -11.940 & 0.932 & 0.400 & 0.466 & -0.79 & 0.310 & 0.033 & 0.292 & 0.105 & 0.430 \\
\hline SD & 0.026 & 0.022 & 0.083 & 0.0268 & 1.225 & 0.109 & 0.041 & 0.044 & 0.18 & 0.015 & 0.005 & 0.019 & 0.015 & 0.011 \\
\hline \multicolumn{15}{|c|}{$\alpha=37^{\circ} .0 .206 \pi$ rad. Mean impact energy $0.42 \mathrm{~J}$} \\
\hline 1 & -1.774 & -1.305 & 0.705 & -0.070 & -5.136 & 0.673 & 0.120 & 0.344 & -1.23 & 0.498 & 0.127 & 0.253 & 0.045 & 0.425 \\
\hline 2 & -1.743 & -1.304 & 0.707 & -0.091 & -5.033 & 0.687 & 0.095 & 0.352 & -1.41 & 0.495 & 0.127 & 0.242 & 0.046 & 0.415 \\
\hline 3 & -1.747 & -1.342 & 0.683 & -0.101 & -3.753 & 0.668 & 0.037 & 0.352 & -0.61 & 0.511 & 0.141 & 0.241 & 0.042 & 0.424 \\
\hline 4 & -1.782 & -1.339 & 0.688 & -0.106 & -8.368 & 0.592 & 0.161 & 0.299 & -1.93 & 0.499 & 0.121 & 0.270 & 0.046 & 0.437 \\
\hline
\end{tabular}




\begin{tabular}{|c|c|c|c|c|c|c|c|c|c|c|c|c|c|c|}
\hline 5 & -1.737 & -1.283 & 0.723 & -0.081 & -6.028 & 0.654 & 0.112 & 0.352 & -2.50 & 0.488 & 0.119 & 0.242 & 0.048 & 0.409 \\
\hline 6 & -1.744 & -1.317 & 0.719 & -0.104 & -2.095 & 0.675 & -0.051 & 0.390 & -0.66 & 0.493 & 0.142 & 0.229 & 0.046 & 0.417 \\
\hline 7 & -1.745 & -1.313 & 0.696 & -0.099 & -5.249 & 0.561 & 0.005 & 0.344 & -1.33 & 0.497 & 0.128 & 0.245 & 0.045 & 0.418 \\
\hline 8 & -1.730 & -1.295 & 0.685 & -0.078 & -7.705 & 0.538 & 0.123 & 0.313 & -1.54 & 0.504 & 0.114 & 0.251 & 0.044 & 0.409 \\
\hline Mean & -1.750 & -1.312 & 0.701 & -0.091 & -5.421 & 0.631 & 0.075 & 0.343 & -1.32 & 0.498 & 0.127 & 0.247 & 0.045 & 0.419 \\
\hline SD & 0.018 & 0.021 & 0.015 & 0.013 & 2.013 & 0.058 & 0.071 & 0.027 & 0.62 & 0.007 & 0.010 & 0.012 & 0.002 & 0.009 \\
\hline \multicolumn{15}{|c|}{$\alpha=60^{\circ} .0 .33 \pi \mathrm{rad}$. Mean impact energy $0.47 \mathrm{~J}$} \\
\hline 1 & -1.191 & -1.981 & -0.220 & -1.419 & 21.944 & 0.410 & -1.761 & 0.331 & -1.07 & 0.579 & 0.197 & 0.068 & 0.204 & 0.469 \\
\hline 2 & -1.196 & -1.993 & -0.122 & -1.316 & 22.731 & 0.516 & -1.649 & 0.430 & -1.14 & 0.630 & 0.234 & 0.064 & 0.178 & 0.476 \\
\hline 3 & -1.214 & -2.016 & -0.197 & -1.491 & 23.818 & 0.481 & -1.822 & 0.387 & -0.96 & 0.517 & 0.192 & 0.066 & 0.226 & 0.484 \\
\hline 4 & -1.205 & -1.950 & -0.225 & -1.557 & 28.597 & 0.595 & -2.033 & 0.478 & -0.97 & 0.400 & 0.148 & 0.054 & 0.256 & 0.458 \\
\hline 5 & -1.199 & -2.001 & -0.070 & -1.227 & 18.533 & 0.459 & -1.504 & 0.374 & -1.03 & 0.685 & 0.254 & 0.074 & 0.149 & 0.477 \\
\hline 6 & -1.213 & -1.996 & -0.263 & -1.482 & 22.244 & 0.338 & -1.870 & 0.297 & -1.02 & 0.542 & 0.185 & 0.071 & 0.222 & 0.478 \\
\hline 7 & -1.177 & -2.012 & -0.138 & -1.367 & 19.757 & 0.447 & -1.600 & 0.353 & -0.99 & 0.620 & 0.222 & 0.069 & 0.184 & 0.475 \\
\hline 8 & -1.189 & -2.003 & -0.272 & -1.379 & 24.042 & 0.466 & -1.687 & 0.338 & -1.63 & 0.680 & 0.221 & 0.063 & 0.201 & 0.485 \\
\hline Mean & -1.198 & -1.994 & -0.188 & -1.405 & 22.708 & 0.464 & -1.741 & 0.373 & -1.10 & 0.582 & 0.206 & 0.066 & 0.203 & 0.475 \\
\hline SD & 0.013 & 0.021 & 0.072 & 0.106 & 3.039 & 0.075 & 0.167 & 0.058 & 0.22 & 0.095 & 0.033 & 0.006 & 0.033 & 0.009 \\
\hline
\end{tabular}

